\title{
Higgs Physics and Supersymmetry
}

\author{
Pran Nath* \\ Department of Physics, Northeastern University, Boston, MA 02115-5005, USA
}

\begin{abstract}
A brief overview of Higgs physics and of supersymmetry is given. The central theme of the overview is to explore the implications of the recent discovery of a Higgs like particle regarding the prospects for the discovery of supersymmetry assuming that it is indeed the spin $0 \mathrm{CP}$ even boson that enters in the spontaneous breaking of the electroweak symmetry. The high mass of the Higgs like boson at $\sim 125 \mathrm{GeV}$ points to the weak scale of supersymmetry that enters in the loop correction to the Higgs boson mass, to be relatively high, i.e., in the $\mathrm{TeV}$ region. However, since more than one independent mass scales enter in softly broken supersymmetry, the allowed parameter space of supersymmetric models can allow a small Higgs mixing parameter $\mu$ and light gaugino masses consistent with $\mathrm{a} \sim 125 \mathrm{GeV}$ Higgs boson mass. Additionally some light third generation sfermions, i.e., the stop and the stau are also permissible. Profile likelihood analysis of a class of SUGRA models indicates that $m_{A}>300 \mathrm{GeV}$ which implies one is in the decoupling phase and the Higgs couplings are close to the standard model in this limit. Thus a sensitive measurement of the Higgs couplings with fermions and with the vector bosons is needed to detect beyond the standard model effects. Other topics discussed include dark matter, proton stability, and the Stueckelberg extended models as probes of new physics. A brief discussion of the way forward in the post Higgs discovery era is given.
\end{abstract}

Keywords: Higgs; Supersymmetry; Unification.

\section{INTRODUCTION}

The Higgs boson [1 3 , is a central part of the standard model of electroweak interactions [4, 5. The long search for it culminated with the observation of a boson in the mass range 125-126 GeV (see Eq.(1)) by the ATLAS [6] and CMS [7] Collaborations using the combined $7 \mathrm{TeV}$ and $8 \mathrm{TeV}$ data (For earlier results from CMS and ATLAS Collaborations which gave a strong indication of a Higgs signal see Refs. [8] and 9]). Pending a more precise determination of its mass we will refer to new particle as $\sim 125 \mathrm{GeV}$ boson. The properties of it still need to be fully established even though there is a strong belief that the $\sim 125 \mathrm{GeV}$ boson is indeed the long sought after Higgs boson of electroweak theory. Here we will first review the current status of the recent developments. Our focus, however, will be on the implications of this discovery for supersymmetry assuming that the newly discovered particle is indeed the Higgs boson. Specifically we will show that the $\sim 125 \mathrm{GeV}$ Higgs boson mass is consistent within SUGRA grand unification [10] which predict an upper limit on the Higgs boson mass of $\sim 130 \mathrm{GeV}$ [1] 13. A $\sim 125 \mathrm{GeV}$ Higgs boson mass puts severe constraints on the parameter space of SUGRA models and it is shown that most of the parameter space consistent with the $\sim 125 \mathrm{GeV}$ Higgs mass lies on the Hyperbolic Branch of radiative breaking of the electroweak symmetry, and specifically on Focal Surfaces. The implications of the $\sim 125 \mathrm{GeV}$ Higgs mass for dark matter is also discussed. Here it is shown that within SUGRA models with universal boundary conditions most of the parameter space of the model lies between the current sensitivity of XENON-100 and the sensitivity of future XENON-1T or SuperCDMS-1T. Analyses within mSUGRA show 14 that the $\sim 125 \mathrm{GeV}$ Higgs mass constraint implies that the CP odd Higgs mass $m_{A}>300 \mathrm{GeV}$ (see also Ref. [15]), and one is in the decoupling limit. In this limit the deviations of the Higgs couplings to matter and to vector fields from the standard model values are expected to be typically small and sensitive measurement of these couplings are needed to discern beyond the standard model effects. A brief discussion is given of the two photon decay of the Higgs which appears to show 1-2 $\sigma$ deviation from the standard model result. An explanation of such deviations would require some light particles in the loops and there are a variety of models that attempt to accomplish that. We also discuss briefly the idea of natural SUSY promoted recently.

There are several other topics discussed in this review. One topic concerns the so called cosmic coincidence which refers to the fact that dark matter to visible matter is in the ratio $\sim 5: 1$. We will discuss how the cosmic coincidence can work in supersymmetric theories. We will also discuss SUSY grand unification specifically regarding the solution to the doublet-triplet splitting in $S O(10)$ using the missing partner mechanism. Other topics discussed within SUSY grand unification include the status of proton lifetime in supersymmetric theories and its current limits

*Electronic address: nath@neu.edu 
from experiment and the radiative breaking of $\mathrm{R}$ parity and how such a breaking can be evaded. We also discuss the (Higgsless) Stueckelberg extensions of the standard model (SM) gauge group and their implications at colliders. Finally we discuss the prospects for the observation of supersymmetry at the LHC.

The outline of the rest of the paper is as follows: In Sec. 2 we discuss the recent developments regarding the discovery of the $\sim 125 \mathrm{GeV}$ boson. In Sec. 3 we discuss the implications of this discovery for supersymmetry and the naturalness of $\mathrm{TeV}$ scalars on the Hyperbolic Branch of radiative breaking of the electroweak symmetry. The other topics discussed in this section are natural SUSY and the decoupling limit. In Sec. 4 we discuss supersymmetry and the cosmic frontier with focus on dark matter. Here we also discuss cosmic coincidence in the context of supersymmetry and show that it results in a two component dark matter. In Sec. 5 we discuss the recent progress on the doublettriplet splitting in $S O(10)$ SUSY grand unified models. A review of the current status of proton stability in SUSY grand unification and the current experimental limits on proton decay lifetimes is given. Radiative breaking of $\mathrm{R}$ parity and the means to avoid such a breaking are also discussed. Sec. 6 describes the Stueckelberg extensions and its relation to strings, and possible tests of such models at colliders. Prospects for SUSY in future data from the LHC are discussed in Sec. 7. In Sec. 8 the way forward after the discovery of the Higgs is discussed.

\section{RECENT DEVELOPMENTS}

The analysis by ATLAS [6] and CMS [7] using the combined $7 \mathrm{TeV}$ and $8 \mathrm{TeV}$ data indicates observation of a signal for a boson with mass

$$
\begin{array}{rr}
\text { CMS : } 125.3 \pm 0.4(\text { stat }) \pm 0.5(\text { sys }) \mathrm{GeV} & 5.0 \sigma \\
\text { ATLAS : } 126.0 \pm 0.4(\text { stat }) \pm 0.4(\text { sys }) \mathrm{GeV} & 5.9 \sigma .
\end{array}
$$

The search channels included $h \rightarrow \gamma \gamma, h \rightarrow Z Z^{*} \rightarrow 4 l, h \rightarrow W W^{*} \rightarrow 2 l 2 \nu$ as well $h \rightarrow b \bar{b}, \tau \bar{\tau}$ with $h \rightarrow \gamma \gamma$ being the dominant discovery channel. One can ask what kind of an object the observed boson is. The two photon final state indicates that it is not a spin one particle due to the Landau-Yang theorem [16. But it could be spin 0 or spin 2. Assuming it is spin 0 one can ask is it a $\mathrm{CP}$ even or a CP odd particle [The spin and CP properties can be determined by analysis of the processes $p p \rightarrow h^{0} \rightarrow Z Z \rightarrow l_{1} \bar{l}_{1} l_{2} \bar{l}_{2}$ [17 and $p p \rightarrow h^{0} \rightarrow W^{+} W^{-} \rightarrow l^{+} l^{-} \nu \bar{\nu}$. See [18] and the references therein.]. Suppose now that it is a CP even particle. In this case, it could be either an ordinary spin 0 particle or a a spin 0 particle that is a remnant of spontaneous breaking which gives mass to gauge bosons, quarks, and leptons [1] [19]. Let us assume that the new boson is a remnant of spontaneous breaking. One may ask what the underlying model is in this case, i.e., is the underlying model the standard model, the supersymmetric standard model, a string model, a composite Higgs model, or something else altogether. Finally assuming that the newly discovered particle is a SUSY Higgs boson one can ask if its properties can shed some light on the breaking of supersymmetry such as if supersymmetry is broken via gravity mediation, gauge mediation, anomaly mediation or something else.

Definitive answers to many of these questions will have to wait for more data. However, partial answers already exist in the data from the Tevatron [20] and from the CMS/ATLAS experiments [6, 7]. For example, there is evidence already that the boson that is discovered is a remnant of spontaneous breaking responsible for giving mass to quarks and leptons. Thus an important test that the scalar boson is responsible for generating mass for fermions is $h_{t}$ (Yukawa) $\sim \frac{m_{f}}{v}$ where $v$ is the VEV of the scalar field. This means that a heavier fermion has a larger branching ratio than a lighter one in the decay of the Higgs. This is consistent with the experimental data from the Tevatron [20] and from the CMS [7] and ATLAS [6] Collaborations where only $b \bar{b}, \tau \bar{\tau}$ are seen but e.g., $\mu^{+} \mu^{-}$is not seen. However, a test of all the couplings will be needed to establish the discovered boson to be a truly standard model like Higgs boson, i.e., a test of the couplings to fermions and to vector bosons specifically the couplings $h b \bar{b}, h t \bar{t}, h \tau \bar{\tau}, h W W, h Z Z, h \gamma \gamma, h Z \gamma$ (see, e.g., Ref. [21]).

Important clues to new physics can emerge by looking at deviations of the Higgs boson couplings from the standard model predictions. In the left panel of Fig. (1) the signal strength $(\mu)$ as given by the ATLAS Collaboration [6 is presented where $\mu$ is defined [6] so that $\mu=0$ corresponds to just the background and $\mu=1$ when one includes the contribution from the standard model Higgs in addition to the background for $m_{h}=126 \mathrm{GeV}$ for various decay final states for the observed boson. The right panel of Fig.(1) gives the analysis by the CMS Collaboration [7. Here $\sigma / \sigma_{S M}$ is plotted where $\sigma$ refers to the production cross section times the relevant branching fractions while $\sigma_{S M}$ is the expectation for the SM case. The possibility that the CMS and ATLAS data may have an excess in the diphoton channel has already led to some excitement. Thus the CMS and ATLAS Collaborations give [6, 7, 

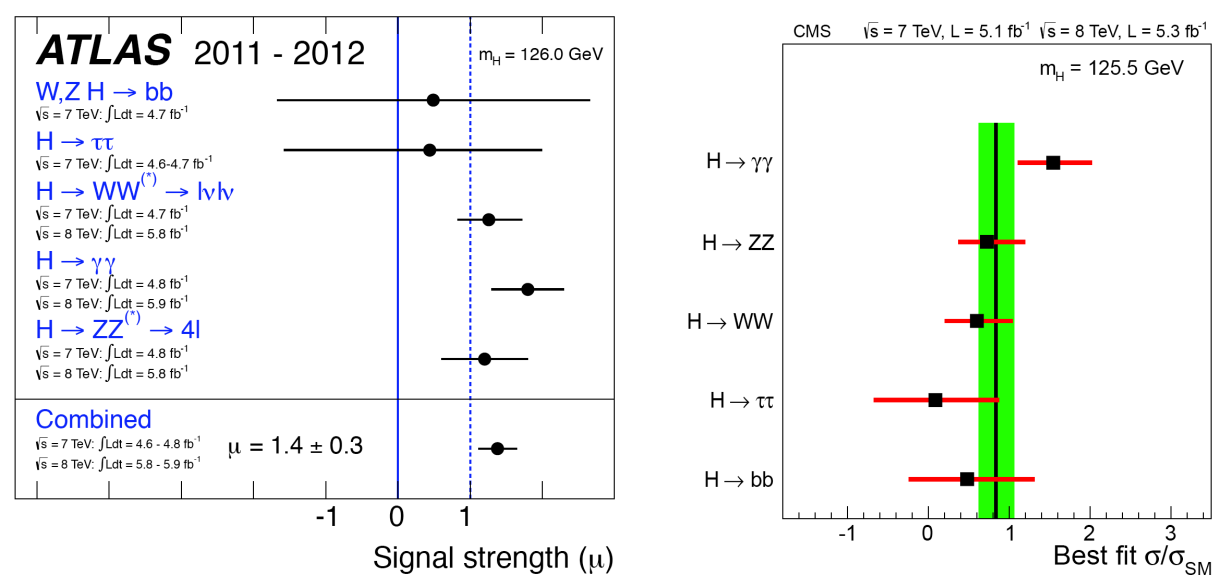

FIG. 1: The left panel gives the analysis by the ATLAS Collaboration 6 for the signal strength parameter $\mu$. The right panel gives the analysis by the CMS Collaboration [7] where $\sigma / \sigma_{S M}$ is plotted, where $\sigma$ refers to the production cross section times the relevant branching fractions while $\sigma_{S M}$ is the expectation for the SM case. Statistical and systematic uncertainties on the evaluation of $\sigma / \sigma_{S M}$ are indicated by horizontal bars which correspond to \pm 1 deviation uncertainties.

$$
\begin{gathered}
\hat{\mu} \equiv \frac{\sigma(p p \rightarrow h)_{o b s}}{\sigma(p p \rightarrow h)_{S M}}=1.4 \pm 0.3(\mathrm{ATLAS}), 0.87 \pm 0.23(\mathrm{CMS}) \\
R_{\gamma \gamma} \equiv \hat{\mu} \frac{\Gamma(h \rightarrow \gamma \gamma)_{o b s}}{\Gamma(h \rightarrow \gamma \gamma))_{S M}}=1.8 \pm 0.5(\mathrm{ATLAS}), 1.6 \pm 0.4(\mathrm{CMS}) .
\end{gathered}
$$

In the SM the Higgs boson can decay into photons via $W^{+} W^{-}$and $t \bar{t}$ in the loops. The $\mathrm{W}$-loop gives the larger contribution which is partially cancelled by the top-loop. To enhance the decay one could add extra particles in the loops (vectors, fermions, scalars) and various works have appeared along these lines in the literature. Specifically, one possibility considered is light particles, e.g., staus or light stops, in the loops [22 24]. One may parametrize the diphoton rate as follows

$$
R_{\gamma \gamma}=\hat{\mu}\left[-1.28\left(1+\delta_{V}\right)+0.28\left(1+\delta_{f}\right)+\delta_{S}\right]^{2} .
$$

The $\delta^{\prime} s$ stand for deviations from the standard model prediction (For a sample of models where such deviations from the standard model are discussed see Refs. 25-29]). An alternative solution is that the excess seen in the diphoton channel is simply a result of QCD uncertainties [30].

\section{IMPLICATIONS FOR SUPERSYMMETRY}

We discuss now the implications of the recent discovery [6, 7] for supersymmetry . From here on we will assume that the discovered boson is indeed the Higgs boson. Now prior to the LHC results of Refs. [6, 7] it was generally thought that the Higgs boson mass could lie between the lower limit given by LEP of $115 \mathrm{GeV}$ and the upper limit which is constrained by partial wave unitarity to be around $800 \mathrm{GeV}$ [31, 32. Inclusion of vacuum stability gives additional more stringent constraints.

The analysis of the vacuum stability is sensitive to corrections beyond the leading order and a recent analysis at the next-to-next -leading order (NNLO) requiring that the vacuum be absolutely stable up to the Planck scale gives 33

$$
m_{h}>129.4+1.4\left(\frac{m_{t}-173.1}{0.7}\right)-0.5\left(\frac{\alpha_{s}\left(M_{Z}\right)-0.1184}{0.0007}\right) \pm 1.0 .
$$

where $m_{h}$ and $m_{t}$ are in $\mathrm{GeV}$. With the inclusion of both the theoretical error in the evaluation of $m_{h}$ estimated at $\pm 1.0 \mathrm{GeV}$ and the experimental errors on the top mass and $\alpha_{s}$ the analysis of Ref. 33 gives

$$
m_{h}>129.4 \pm 1.8 \mathrm{GeV},
$$


for the standard model to have vacuum stability up to the Planck scale. This excludes the vacuum stability for the $\mathrm{SM}$ for $m_{h^{0}}<126 \mathrm{GeV}$ at the $2 \sigma$ level 33 . Thus the observation of a Higgs mass of $\sim 125 \mathrm{GeV}$ would give vacuum stability up to only scales between $10^{9}-10^{10} \mathrm{GeV}$ and stability up to the Planck scale would require new physics (see, however, Refs. 34, 35]). Such new physics could be supersymmetry but other models can be found that can also achieve such a result (for recent examples see Refs [36, 37]). However, there still remains the well known hierarchy problem, i.e., the standard model gives large loop corrections to the Higgs boson mass

$$
m_{h}^{2}=m_{0}^{2}+O\left(\Lambda^{2}\right)
$$

Since $\Lambda \sim O\left(10^{16}\right) \mathrm{GeV}$ a large fine tuning is involved to get the Higgs mass in the electroweak region. The SUSY version of SM is free of the large fine tuning problems. Thus MSSM has no large hierarchy problem and gives perturbative physics up to the GUT scale. In SUSY the Higgs boson mass at tree level is $<M_{Z}[38$ and loop corrections are needed to lift it above $M_{Z}$. The dominant one loop contribution arises from the top-stop sector and is given by 39 , 40 ]

$$
\Delta m_{h^{0}}^{2} \simeq \frac{3 m_{t}^{4}}{2 \pi^{2} v^{2}} \ln \frac{M_{\mathrm{S}}^{2}}{m_{t}^{2}}+\frac{3 m_{t}^{4}}{2 \pi^{2} v^{2}}\left(\frac{X_{t}^{2}}{M_{\mathrm{S}}^{2}}-\frac{X_{t}^{4}}{12 M_{\mathrm{S}}^{4}}\right)+\cdots,
$$

where $v=246 \mathrm{GeV}(v$ is the Higgs $\mathrm{VEV}), M_{\mathrm{S}}$ is an average stop mass, and $X_{t}$ is given by

$$
X_{t} \equiv A_{t}-\mu \cot \beta
$$

The loop correction is maximized when $X_{t} \sim \sqrt{6} M_{\mathrm{S}}$. It should be noted that when we talk about supersymmetry we are actually taking about local supersymmetry [41, 42] or supergravity 43,45] since model building makes sense only in such a framework. This is so because breaking of supersymmetry would require the cancellation of the vacuum energy. This can happen in models based on supergravity because the scalar potential is not positive definite and it is possible to fine tune the vacuum energy to zero. Alternately there may be stringy mechanisms for the vacuum energy to be be small [46]. Further, it should be noted that since supergravity is the field point limit of strings an analysis within the framework of supergravity can provide a wide class of models including those arising from strings (such as, e.g., 47, 48) . Supergravity grand unified models 10, 49 provide a reliable framework for the exploration of physics from the electroweak scale up to the GUT scale of $\sim 10^{16} \mathrm{GeV}$. Under the assumption of universal boundary conditions the soft breaking sector of this model gives a very constrained set of soft terms [10, 49, [50] (for a review see Ref. [51]). Specifically the soft breaking sector of this theory after radiative breaking of the electroweak symmetry is given by the set of parameters $m_{0}, m_{1 / 2}, A_{0}, \tan \beta$ and the sign of the Higgs mixing parameter $\mu$. In this model usually referred to as mSUGRA or CMSSM, the upper limit on the Higgs mass is $m_{h} \sim 130 \mathrm{GeV}$ [11 13]. This result is to be contrasted with the more general result in SUSY models where one expects the Higgs mass to lie below $\sim 150$ $\mathrm{GeV}$ [52. The upper limit predicted in mSUGRA is exhibited in Fig. (2). It is meaningful that at the end of the day the Higgs mass ended up below the upper limit predicted in mSUGRA. This provides a positive signal that what we are seeing is a SUSY Higgs. The implications of the new results [6, 7] have been analyzed in a number of works [28, 53,70$]$.

\section{A. Natural TeV Size Scalars}

TeV size scalars arise naturally in SUGRA models with radiative breaking of the electroweak symmetry. Thus the radiative breaking of the electroweak symmetry allows a determination of the Higgs mixing parameter $|\mu|$ such that

$$
\begin{array}{r}
\mu^{2}=-\frac{1}{2} M_{Z}^{2}+\frac{\left(m_{H_{1}}^{2}+\Sigma_{1}\right)-\left(m_{H_{2}}^{2}+\Sigma_{2}\right) \tan ^{2} \beta}{\left(\tan ^{2} \beta-1\right)} \\
\Sigma_{a}=v_{a}^{-1} \frac{\partial \Delta V}{\partial v_{a}}, a=1,2,
\end{array}
$$

where $v_{a}=\left\langle H_{a}>\right.$ and $H_{2}\left(H_{1}\right)$ give masses to the up quarks (down quarks and leptons) and $\Sigma_{a}(a=1,2)$ are radiative corrections at the one loop level [73. To display the dependence of $\mu^{2}$ on the soft parameters more explicitly one may write Eq. 10 so that

$$
\mu^{2}+\frac{1}{2} M_{Z}^{2}=m_{0}^{2} C_{1}+A_{0}^{2} C_{2}+m_{\frac{1}{2}}^{2} C_{3}+m_{\frac{1}{2}} A_{0} C_{4}+\Delta \mu_{\text {loop }}^{2}
$$




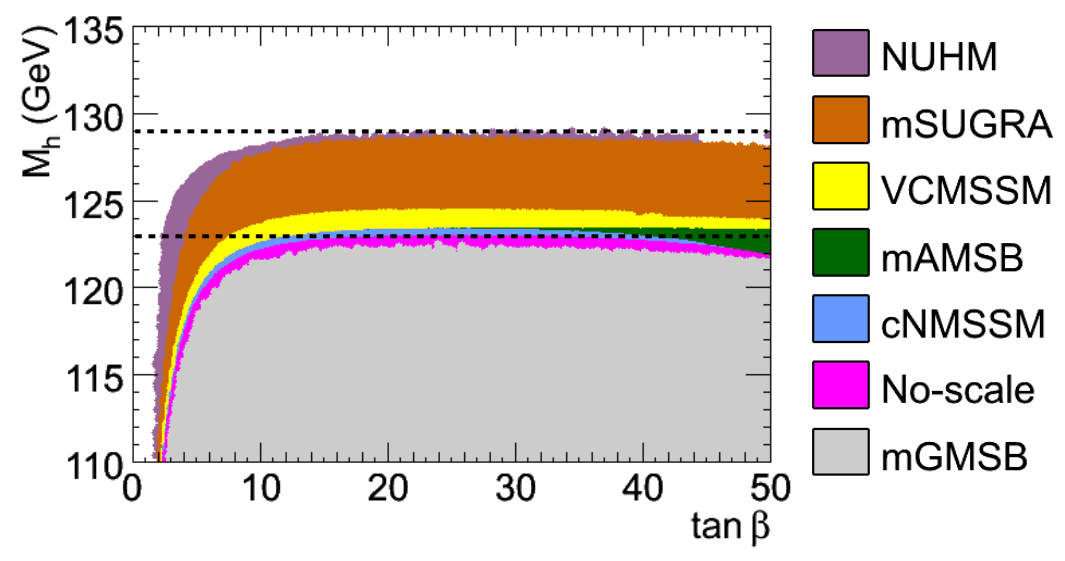

FIG. 2: An analysis of the ranges of the Higgs boson mass vs $\tan \beta$ for a variety of different models as given in Arbey et.al. [12, 71. The upper limit on $M_{S}$ is chosen to be $3 \mathrm{TeV}$. The figure is taken from Ref. [12].
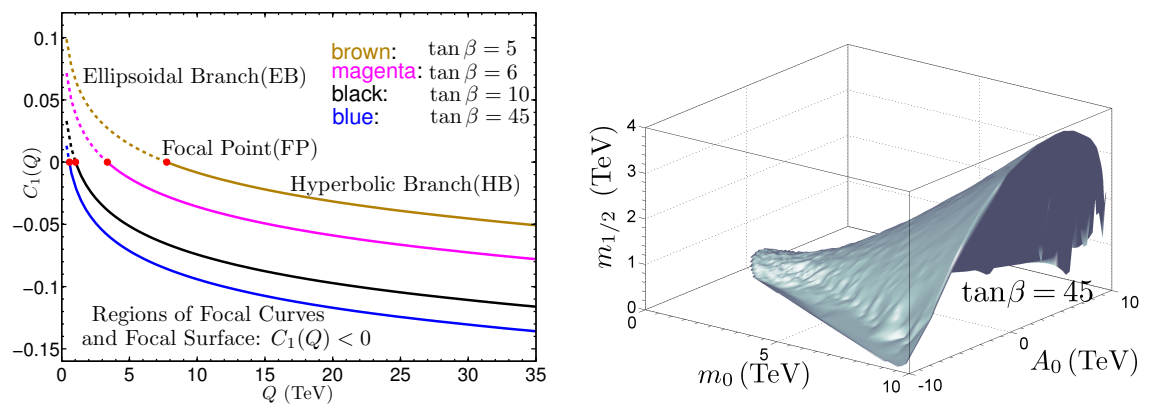

FIG. 3: Left panel: $C_{1}(Q)$ as a function of $Q$ for $\tan \beta=5$ (brown), $\tan \beta=6$ (magenta), $\tan \beta=10$ (black) and $\tan \beta=$ 45 (blue). Right panel: A Focal Surface for the case and $\tan \beta=45$ while $m_{0}, m_{1 / 2}, A_{0}$ can all get large while $\mu$ remains fixed so that $\mu=(0.465 \pm 0.035) \mathrm{TeV}$. Figs. taken from Ref. 72.

where $C_{1}-C_{4}$ depend on $\tan \beta$ but do not have an explicit dependence on the soft parameters $m_{0}, m_{1 / 2}, A_{0}$, while $\mu_{\text {loop }}^{2}$ has a complicated dependence on soft parameters since it arises from a loop contribution. One may write Eq. 11 . as

$$
\mu^{2}+\frac{1}{2} M_{Z}^{2}=m_{0}^{2} C_{1}+\bar{A}_{0}^{2} C_{2}+m_{\frac{1}{2}}^{2} \bar{C}_{3}+\Delta \mu_{\text {loop }}^{2},
$$

where $\bar{A}_{0}$ and $\bar{C}_{3}$ are simply related to $A_{0}, m_{1 / 2}, C_{2}, C_{3}, C_{4}$, so that $\bar{A}_{0}=A_{0}+\left(C_{4} / 2 C_{2}\right) m_{1 / 2}$ and $\bar{C}_{3}=C_{3}-C_{4}^{2} /\left(4 C_{2}\right)$. The functions $C_{1}-C_{4}$ and $\Delta_{\text {loop }}^{2}$ are functions of the renormalization group scale $Q$, and the constraint of Eq. 12 become more transparent if one goes to the scale $Q_{0}$ where the loop correction $\Delta_{\text {loop }}^{2}$ is minimized so one may examine the remaining terms to elucidate the constraint. Here one typically finds that $C_{2}, \bar{C}_{3}$ are always positive, but $C_{1}$ could be either positive, vanishing or negative. These cases lead to drastically different constraints on the allowed parameter space due to radiative breaking of the electroweak symmetry.

The case $C_{1}>0$ implies that the soft parameters lie on the surface of an ellipsoid for fixed $\mu$ and so this branch of radiative breaking of the electroweak symmetry may appropriately be called the Ellipsoidal Branch (EB) [74. In this case none of the soft parameters can get large for a fixed $\mu$. The case where $C_{1} \leq 0$ will be called the Hyperbolic Branch 74 76. Here one, two or more soft parameters can get large while $\mu$ can remain small. The case when $C_{1}=0$ is the one where one soft parameter can get large, i.e., $m_{0}$ with no visible effect on $\mu$ as can be seen from Eq. (12). One may label this as the Focal Point (HB/FP) (it is closely related to the Focus Point of Ref. 77]) and it is in fact, the transition point between the branches $\mathrm{EB}$ and $\mathrm{HB}$. The case when $C_{1}<0$ and two parameters can get large 
while $\mu$ remains fixed constitutes the Focal Curve region of the Hyperbolic Branch. There are actually two types of Focal Curves: those where $m_{0}, \bar{A}_{0}$ get large which we label as $\mathrm{HB} / \mathrm{FC} 1$ and those where $m_{0}, m_{\frac{1}{2}}$ get large with $\mu$ fixed which we label as HB/FC2. Focal Curves HB/FC1 typically have the asymptotic property [78] that $\left|\bar{A}_{0} / m_{0}\right| \simeq 1$ when $\mu$ is fixed and $m_{1 / 2}$ is relatively small compared to $m_{0}$. The case where all the dimensioned soft parameters, i.e., $m_{0}, m_{1 / 2}, \bar{A}_{0}$, get large constitutes the Focal Surface region of the Hyperbolic Branch. Thus the Hyperbolic Branch contains Focal Points, Focal Curves and Focal Surfaces. The left panel of Fig.(3) gives a pictorial classification of the Ellipsoidal Branch, and of the Hyperbolic Branch. Plotted in Fig. $(3)$ is $C_{1}(\vec{Q})$ as a function of $Q$. The dotted curves identify the Ellipsoidal Branch region, and the solid curves the Hyperbolic Branch region while the dots are the Focal Points which form the boundary between the Ellipsoidal Branch and the Hyperbolic Branch. The right panel of Fig.(3) gives a display of a Focal Surface which exhibits regions of the parameter space of mSUGRA where two or more soft parameters among $m_{0}, m_{1 / 2}, A_{0}$ get large while $\mu$ remains small. In the top left panel of Fig. (4) we exhibit the allowed parameter space of mSUGRA under the current experimental constraints. Here one finds that most of the parameter space of mSUGRA lies on focal surfaces which, of course, include focal curves.

\section{B. Natural SUSY}

Often a criterion of fine tuning is imposed to discriminate among models. A conventional choice is [79]

$$
f=\max \frac{\partial \ln m_{h^{0}}^{2}}{\partial \ln \alpha_{i}}
$$

where $\alpha_{i}$ are the basic parameters on which $m_{h^{0}}^{2}$ depends. In the decoupling limit in MSSM on has

$$
m_{h^{0}}^{2}=M_{Z}^{2} \cos ^{2} 2 \beta+\Delta m_{h^{0}}^{2}
$$

where $\Delta m_{h^{0}}^{2}$ is given by Eq. (8). For large $\tan \beta$ the loop correction needed is rather substantial to raise the Higgs boson mass from the tree level value to the experimentally observed value of $m_{h^{0}} \sim 125 \mathrm{GeV}$. In MSSM the scale $M_{s}$ is in the $\mathrm{TeV}$ region implying a large average stop mass, and Eq. 13 then leads to a fine tuning parameter $f$ of size $O(100)$ or larger. The level of fine tuning could be reduced if there are additional sources to the Higgs boson mass term which might arise from F term quartics (see, e.g., Ref. [38, 80, 81]) or D term quartics (see, e.g., Refs. [82, 83]). In this case a smaller loop correction would be needed leading to a smaller level of fine tuning. As an example the F term quartics arise in NMSSM where one includes a singlet field $\mathrm{S}$ and a coupling among the doublets and the singlet field of the form $W \supset \lambda S H_{1} H_{2}$. Here at the tree level Higgs mass ${ }^{2}$ contains an additional term $81 \lambda^{2} v^{2} \sin ^{2} 2 \beta$. A significant contribution from this sector requires a large $\lambda$ and a small $\tan \beta$. However, $\lambda$ is constrained to lie below 0.7 otherwise the scale above which non-perturbativity sets is lies below the grand unification scale. In this set up the stop masses can lie in the few hundred GeV region and the fine tuning is significantly reduced [84. However, the downside of including a singlet is well known (see, e.g., Refs. [85, 86]). A variation of this idea is $\lambda$ SUSY [84]. Here one considers $\lambda$ larger than 0.7 since that would further reduce the fine tuning and values of $\lambda$ as large as 2 are considered. The upper limit $\lambda=2$ is taken so that the cutoff scale above which non-perturbativity sets is does not lie below $10 \mathrm{TeV}$ so not to disturb precision electroweak physics. There are several other versions of natural SUSY (see, e.g., [87, 88]). Mostly these are effective theories valid up to around $10 \mathrm{TeV}$ and are not necessarily UV complete.

A more generic statement of natural SUSY is that the particles that enter in the solution to the hierarchy problem for the Higgs should be light while the others could be heavy. Thus the particles required to be light are

$$
\left(\tilde{t}_{L}, \tilde{b}_{L}\right), \tilde{t}_{R}, \tilde{H}_{2}, \tilde{H}_{1}
$$

along with the Higgses. It is possible that very low mass stops could have been missed in the LHC analyses and thus it is necessary to pursue this possibility to close any existing holes in signature analyses for supersymmetry (for a broad search strategy for natural SUSY see, Refs. [89, 90]). The current experimental constraints on the stop mass from the ATLAS analysis are exhibited in Fig.(5) in the neutralino vs stop mass plane (see also Ref. 91]). The white regions in Fig. (5) are the ones not excluded by the current data. Specifically one finds that there is a white region around stop mass of $200 \mathrm{GeV}$ which is yet not excluded by the experimental constraints. It is hoped that this region will be probed by the additional data which will soon be forthcoming from the ATLAS and from the CMS detectors at $\sqrt{s}=8 \mathrm{TeV}$. Exclusion of the remaining low stop mass region will make the natural SUSY idea not sustainable. 
It should be noted that the choice of criteria one uses to quantify naturalness in the context of electroweak physics are rather subjective and different choices can lead to widely different results. Since the point of contact between theory and the LHC is the existence of light particles which can be searched at the LHC, a pragmatic approach is to simply explore the allowed parameter space of models which would lead to light particles consistent with a $\sim 125$ GeV Higgs boson mass constraint without prejudice. In the framework of high scale models, one could accomplish this in a variety of ways. For instance, with universal boundary conditions one can have a $\sim 125$ GeV Higgs boson mass with large $m_{0}$ but light gaugino masses with a relatively small $m_{1 / 2}$ and some light third generation particles can arise with a large trilinear coupling. The other possibility is use of non-universalities both in the scalar sector (see, e.g., Refs. 92, 93) and also in the gaugino sector (see, e.g., Ref. 94) to look for a light SUSY mass spectrum. (For a recent work in a top-down approach which examines the range of GUT parameters which leads to a natural SUSY spectrum see Ref. 93]). Here we mention that solutions of this type arise on the Hyperbolic Branch (on Focal Points, Focal Curves and Focal Surfaces) as discussed earlier in this section. In these regions $m_{0}$ is typically large, which has the added benefit that a large $m_{0}$ helps suppress SUSY FCNC and SUSY CP problems along with the fact that large scalar masses help in stabilizing the proton from lepton and baryon number violating dimension five operators. It has also been proposed that a more realistic way to look at fine tuning is to consider not only the the EWSB fine tuning but a composite fine tuning parameter which also includes the fine tuning from other sectors as well such as the flavor constraints [95].

\section{SUSY Higgs in the decoupling phase}

The deviations of the MSSM light Higgs boson from its standard model couplings are often characterized in terms of decoupling and non-decoupling limits [96, 97]. Often the decoupling limit is defined by $\cos ^{2}(\beta-\alpha)<0.05$, where $\alpha$ is the mixing angle between the two CP-even Higgses of MSSM, i.e., $H_{1}^{0}, H_{2}^{0}$ to produce the mass eigenstates $h^{0}, H^{0}$, and is typicallly realized when $M_{A}>300 \mathrm{GeV}$. Using the profile likelihood analysis (see, e.g., Refs. [98, 99]) the work of Ref. [14] gives $m_{A}>300 \mathrm{GeV}$ under the constraint of $m_{h^{0}} \sim 125 \mathrm{GeV}$ so one is in a decoupling phase. The decoupling phase also explains the experimental limits from LHCb (http://inspirehep.net/record/1094383) on $B_{s}^{0} \rightarrow \mu^{+} \mu^{-}$being very close to the SM value, since the SUSY contribution proceeds via the diagram involving the exchange of neutral Higgses [100 106]. Thus the most recent result from LHCb gives $B r\left(B_{s}^{0} \rightarrow \mu^{+} \mu^{-}\right)<4.5 \times 10^{-9}$ while the standard model result is $\operatorname{Br}\left(B_{s}^{0} \rightarrow \mu^{+} \mu^{-}\right) \sim 3.2 \times 10^{-9}$ implying the supersymmetric contribution to this process is small which is consistent with the decoupling limit (for implications of the $\operatorname{Br}\left(B_{s}^{0} \rightarrow \mu^{+} \mu^{-}\right.$) constraint on dark matter see Refs. [104, 107]). In the analysis carried out in Ref. [14] one finds that while the first two generation squarks are typically heavy several other sparticles can be relatively light. These include the gluino, the chargino, the neutralino, the stop, the stau. Further, the $\mu$ parameter can be light. In the top right panel of Fig. (4) we give an analysis of the gluino mass $m_{\tilde{g}}$ vs $\mu$ under the constraint of the $\sim 125 \mathrm{GeV}$ Higgs mass. One finds that there are significant regions of the parameter space (see the lower left corner of the top right panel of Fig. (4)) where $\mu$ is small, typically $O(200) \mathrm{GeV}$ and the gluino is also relatively light, i.e., typically around a TeV. This region appears to be the prime region for the exploration at the next round of LHC experiment. An analysis of signatures for the MSSM Higgs boson in the non-decoupling region at the LHC is given in Ref. 108.

Returning to the decoupling limit the deviations of the Higgs boson couplings to fermions and to vector bosons from the SM values are expected to be typically small in the decoupling limit. Thus the couplings of Higgs to the fermions and to the vector bosons at the tree level have the form

$$
L_{h^{0} A \bar{A}}=-\frac{m_{f}}{v} h^{0} f \bar{f}-\frac{2 M_{W}^{2}}{v} h^{0} W^{\mu+} W_{\mu}^{-}-\frac{M_{Z}^{2}}{v} h^{0} Z^{\mu} Z_{\mu}+\cdots
$$

To test beyond the standard model (BSM) physics one may define the tree level couplings the ratio

$$
R_{h^{0} A \bar{A}}=g_{h^{0} A \bar{A}}^{\mathrm{BSM}} / g_{h^{0} A \bar{A}}^{\mathrm{SM}},
$$

where

$$
R_{h^{0} A \bar{A}}=1+\Delta_{A}
$$

and $\Delta_{A}$ encode the BSM contribution. Thus accurate measurements of $\Delta_{A}$ are needed to discern BSM physics. How to extract the Higgs bosom couplings from LHC data has been discussed by a number of authors (see, for example, 
Refs. 109 112]). A post Higgs discovery analysis on the Higgs couplings using LHC data is given in Ref. 113] and exhibited in the bottom left panel of Fig.(4) where the central values and the errors on the couplings are displayed. The analysis of Ref. [113] finds no significant deviation from the standard model in any of the Higgs couplings. However, the errors in the determinations of the couplings are rather significant. One can ask how accurately we will be able measure these couplings eventually at the LHC? According to the analysis of Peskin [114 at LHC with $\sqrt{s}=14 \mathrm{TeV}$, and $\mathcal{L}=300 \mathrm{fb}^{-1}$ one could achieve an accuracy in the determination of the Higgs couplings in the range 10-20\% which would broadly test the standard model. However, it is unlikely that LHC can achieve an accuracy at the level of $5 \%$ which may be needed to discriminate Higgs bosons of new physics models from the Higgs boson of the standard model. Better accuracies could be achieved at the ILC according to the analysis of Ref. 114. This is exhibited in the bottom right panel of Fig. (4). Thus it is estimated that an ILC with $1000 \mathrm{fb}^{-1}$ of integrated luminosity at $\sqrt{s}=1 \mathrm{TeV}$ should be able to reach a sensitivity of about $3 \%$ for all the Higgs couplings [114. Such a measurement will obviously be of considerable value in the discovery of new physics beyond the standard model.

In the standard model the electro-weak corrections to $a_{\mu}=\left(g_{\mu}-2\right) / 2$ arise from the exchange of the $W$ and of the $Z$ boson. In supersymmetric theories the contribution to $g_{\mu}-2$ can arise from the exchange of charginos-sneutrinos and of neutralinos-smuons. These contributions at the one loop level were computed in [115, 116]. Obviously the supersymmetric contribution depends sensitively on the sparticle spectrum. The experimental determination of $a_{\mu}$ depends heavily on the hadronic corrections. These corrections are estimated by using $e^{+} e^{-}$annihilation cross section and by using the $\tau$ decays. The analysis using $e^{+} e^{-}$annihilation gives $\delta a_{\mu}=(28.7 \pm 8.0) \times 10^{-10}(3.6 \sigma)$ while for $\tau$-based hadronic contributions one has $\delta a_{\mu}=(19.5 \pm 8.3) \times 10^{-10}(2.4 \sigma)$ [117, 118]. A more recent analysis using a complete tenth-order QED contribution [119] and the hadronic error analysis based on $e^{+} e^{-}$annihilation gives $\delta a_{\mu}=24.9(8.7) \times 10^{-10}(2.9 \sigma)$ deviation. Using one loop supersymmetric contribution to $a_{\mu}$, either experimental result would give some tension in SUSY analyses where the sfermion spectrum is heavy. However, estimates of two loop effects could reduce this tension [120. Alternately part of the Higgs mass correction could arise from extra matter [121? -125 or through D terms arising from extra gauge groups under which the Higgs boson could be charged (see, e.g., Ref. [127]).

\section{SUPERSYMMETRY AND THE COSMIC FRONTIER}

Supersymmetry enters in many aspects of the cosmic frontier. These include (i) dark matter, (ii) dark energy, (iii) inflation, and (iv) baryogenesis. We focus here on dark matter. In supersymmetric theories with $\mathrm{R}$ parity, the lightest R parity odd particle (LSP) is stable, and if such a particle is neutral it can be a possible candidate for dark matter. In SUGRA unified models, over much of the parameter space of models, the neutralino turns out to be the LSP [135] and thus the neutralino becomes a candidate for dark matter with $\mathrm{R}$ parity. It is then reasonable to ask what the implications of the discovery of the Higgs boson with a mass of $\sim 125 \mathrm{GeV}$ are on dark matter. It turns out that the $\sim 125 \mathrm{GeV}$ Higgs mass puts rather severe constraints on the allowed parameter space and thus on the allowed range of the spin independent cross section [11, 14] as exhibited in Fig. 66 for the mSUGRA model. Here one finds that much of the allowed parameter space of the model lies within the current XENON-100 limit [129 132 and the limit that one will be able to achieve with XENON-1T [133] and with superCDMS [134] in the future. An analysis of the gaugino-higgsino content of the models analyzed shows that the allowed model points consistent with all constraints have a significant Higgsino content. We discuss now another aspect of dark matter which relates to cosmic coincidence as discussed below.

There is an interesting cosmic coincidence in that 136.

$$
\frac{\Omega_{D M} h_{0}^{2}}{\Omega_{B} h_{0}^{2}}=4.99 \pm 0.20
$$

where $\Omega_{D M} h_{0}^{2}$ is the dark matter relic density and $\Omega_{B} h_{0}^{2}$ is the relic density of baryonic matter. The above appears to indicate that the two are somehow related. One proposal is that dark matter is generated by the transfer of a net $B-L$ created in the early universe to the dark matter sector. (For a recent work see [137] and for a review see [138]). There are two main issues to address for the mechanism to work. The first concerns how to transfer $(B-L)$ generated in the visible sector in the early universe to the dark matter sector and the second is how to dissipate thermal dark matter. Regarding the first one can do so by considering a $B-L$ transfer equation of the form $L_{i n t}=\frac{1}{M^{3}} \psi^{3} L H$. When the transfer interaction is in equilibrium, one can solve for $\Omega_{D M} / \Omega_{B}$ using the thermal equilibrium technique [139, 140] which gives $\frac{\Omega_{D M}}{\Omega_{B}}=\frac{X \cdot m_{X}}{B \cdot m_{B}}$. where $X$ is the number of dark matter particles, and $B$ 

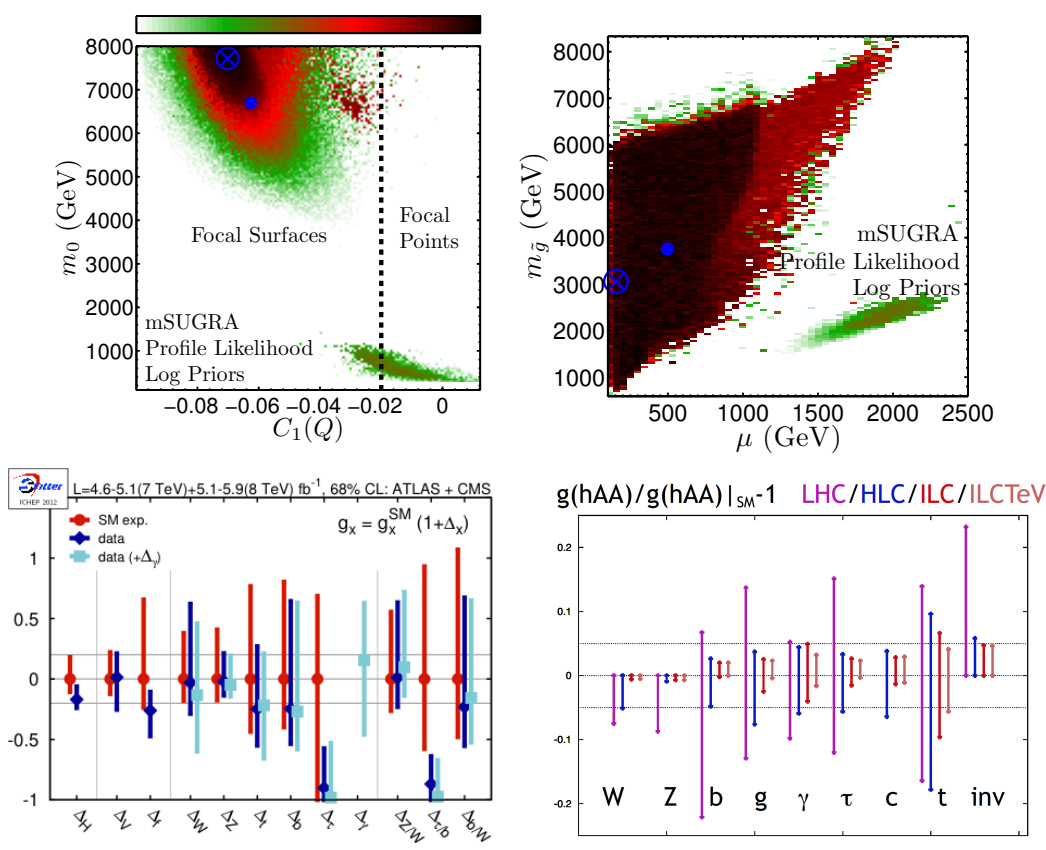

FIG. 4: Top left: A profile likelihood analysis of the parameter space of mSUGRA model which exhibits the dispersion in $m_{0}$ as a function of $C_{1}(Q)$. The analysis shows that the most of the allowed parameter space consistent with all constraints lies on focal surfaces. The figure is taken from Akula et.al. 128. Top right: A profile likelihood analysis in the gluino mass vs the Higgs mixing parameter $\mu$. The analysis shows that there are regions with small $\mu$, which may be construed as natural, where the gluino can also be relatively light. The figure is taken from Akula et.al. 14. Bottom left: Extraction of the Higgs boson couplings using the LHC data. The analysis shows no significant deviations from the standard model prediction. However, the extraction also shows significant uncertainties. The figure is taken from Plehn et.al. [113. Bottom right: A comparison of the accuracy with which LHC can determine the Higgs couplings to quarks and leptons and to vector bosons at $\sqrt{s}=14$ TeV and $L=300 \mathrm{fb}^{-1}$ and the accuracy with which ILCTeV can determine the couplings at $\sqrt{s}=1 \mathrm{TeV}$ and $1000 \mathrm{fb}^{-1} \mathrm{of} \mathrm{integrated}^{-1}$ luminosity. The figure of this panel is taken from Peskin [114.

is the number of baryons and $m_{X}, m_{B}$ are their corresponding masses. Regarding the second item, i.e., thermal dark matter, one needs an efficient mechanism for its dissipation. Additionally, an important issue regarding fermionic dark matter relates to the possibility that such matter can acquire Majorana masses allowing it to oscillate and dissipate. This issue needs to be addressed as well.

Cosmic coincidence can work in SUSY models with R parity with an extended gauge group. For example, one may consider an extension of the conventional SUGRA models with the gauge group $S U(3)_{C} \times S U(2)_{L} \times U(1)_{Y}$ with an extra $U(1)_{X}$ gauge factor. A convenient choice is $X=B-L$ since $B-L$ is not washed out by spharelon interactions. Specifically, let us suppose we consider a transfer equation so that $W=\frac{1}{M^{3}} X^{2}\left(L H_{u}\right)^{2}$. We assume that the $U(1)_{X}$ gauge boson gets its mass via the Stueckelberg mechanism [141 145. Here there are two dark matter particles, i.e., $\psi_{X}$ (which carries a lepton number) and the conventional dark matter particle of supersymmetry, i.e., the neutralino $\tilde{\chi}^{0}$. Thus the total relic density in this case is 146.

$$
\Omega_{\mathrm{DM}}=\Omega_{\mathrm{DM}}^{\mathrm{asy}}+\Omega_{\mathrm{DM}}^{\mathrm{sym}}+\Omega_{\tilde{\chi}^{0}} .
$$

Cosmic coincidence can work if one assumes that $\psi_{X}$ is the dominant component (around 90\%) and the neutralino is the subdominant component (around $10 \%$ ). $\Omega_{\mathrm{DM}}^{\text {sym }}$ can be dissipated by rapid annihilation via a Breit-Wigner $Z_{X}^{\prime}$ pole. An interesting possibility to investigate is if the neutralino which is a subdominant component in the relic density is still detectable. This appears to be the case in an explicit model constructed [146] where the dark matter carries a gauged quantum number which does not allow Majorana mass for dark fermion, and thus fermionic dark matter does not oscillate and is not washed out. We note in passing that an interesting new development in the analysis of dark matter is the idea of dynamical dark matter (DDM) [147, 148. In the DDM scheme the dark matter in the universe consists of a large number of components which interact with each other and have different masses and abundances [147, 148. Thus in this case one has multi-component dark matter consisting of an ensemble than than one species. 


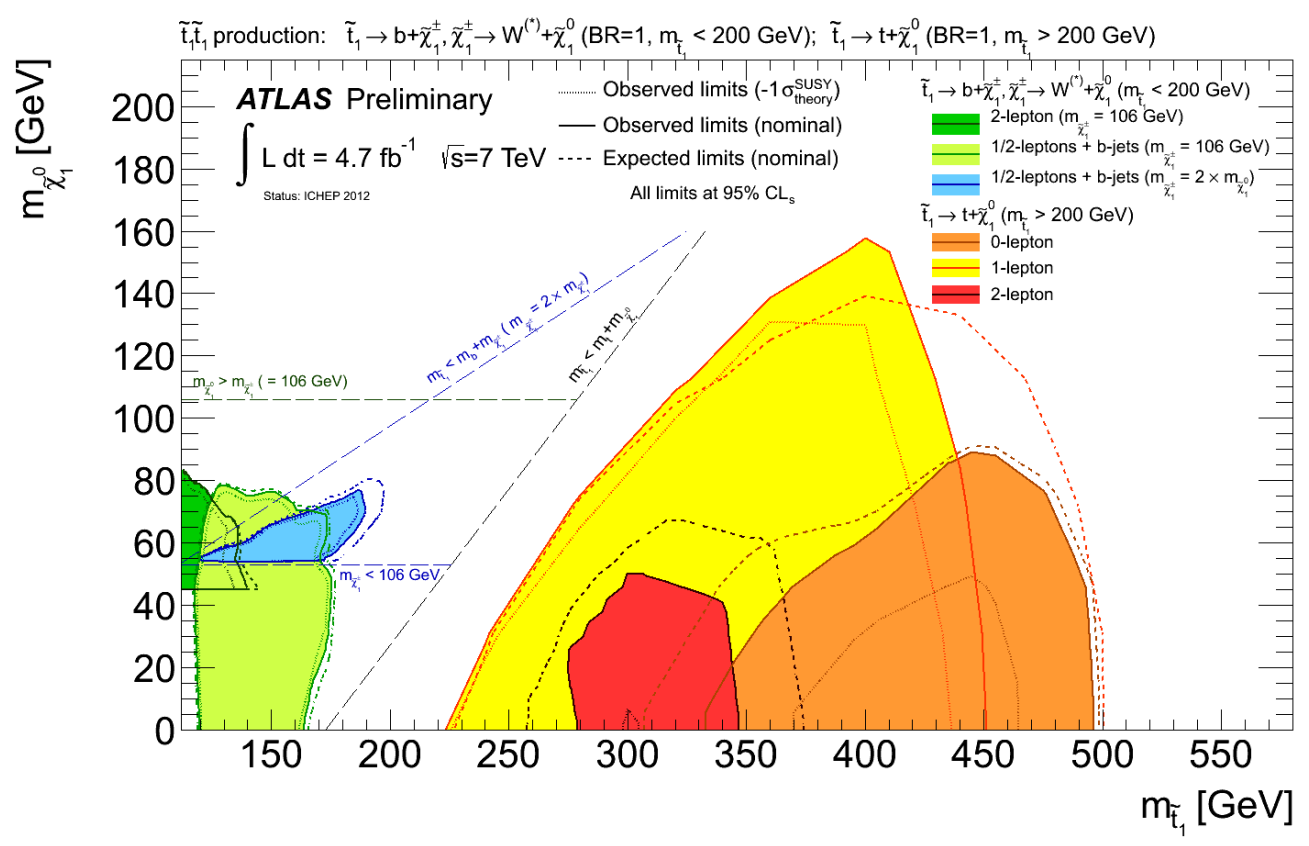

FIG. 5: An exhibition of the excluded region in the neutralino $\tilde{\chi}_{1}^{0}$ and stop mass plane. The low stop mass (white) region around $200 \mathrm{GeV}$ is not yet excluded. Figure taken from https://twiki.cern.ch/twiki/bin/view/AtlasPublic/SupersymmetryPublicResults.

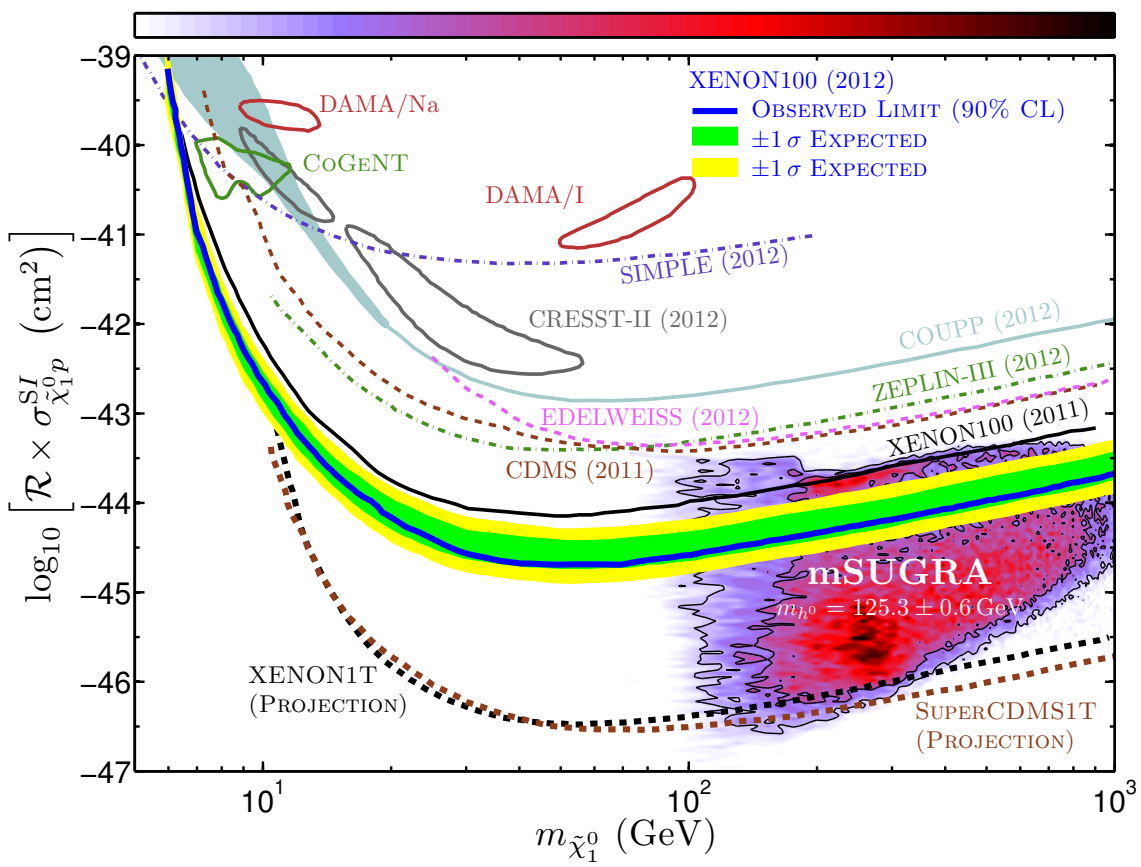

FIG. 6: An analysis of the spin-independent neutralino-proton cross section exhibited as a function of the neutralino mass for the model mSUGRA including the $\sim 125 \mathrm{GeV}$ Higgs mass constraint [128. It is seen that most of the parameter space lies between the current XENON100 limit [129 132] and the limit from XENON-1T [133] and from SuperCDMS-1T [134. 


\section{SUSY GRAND UNIFICATION}

SUSY $S O(10)$ models, as usually constructed, have two drawbacks, both related to the symmetry breaking sector. The first is that more than one mass scale are used in breaking of the GUT symmetry: one to reduce the rank, and the other to reduce the symmetry all the way down to the SM gauge group. Thus one uses $16+\overline{16}$ or $126+\overline{126}$ for rank reduction and uses 45, 54 or 210 for breaking the symmetry down to the standard model gauge symmetry. Finally one uses 10 plets for breaking the electroweak symmetry. Now multiple step breaking requires additional assumptions relating VEVs of the different breakings to explain gauge coupling unification of the electroweak and the strong interactions. A single step breaking does not require such an assumption [149, 150]. The second issue which is generic to grand unified models is the doublet-triplet problem, which is how to make the Higgs triplets heavy while keeping the Higgs doublets light. Regarding the first a single step breaking can be achieved by using $144+\overline{144}$ Higgs to break the GUT symmetry. Regarding the doublet-triplet splitting there are a variety of ways in which one can accomplish this. One of these is the missing partner mechanism [151, 152. In SU(5) this is accomplished by using a 75 plet of Higgs fields to break the GUT symmetry, while the matter sector consists of $5+\overline{5}$ of light Higgs fields and a $50+\overline{50}$ of superheavy Higgs fields where $50+\overline{50}$ have the property that they contain one triplet and anti-triplet pair but no doublet pair. Allowing for mixings between the light and the heavy heavy Higgs fields one finds that all the triplets and anti-triplets become heavy while the Higgs doublets remain light 151, 152. Recently, one example of the missing partner mechanism in $S O(10)$ was found in Ref. [153] which was anchored in $126+\overline{126}$ of Higgs fields. In Ref. [154] an exhaustive treatment of the missing partner mechanism is given. Here two additional cases were found which are anchored in $126+\overline{126}$ of Higgs while one unique case is found which is anchored in $560+\overline{560}$ [154]. In the latter case one can simultaneously break the gauge symmetry at one scale and also achieve a natural doublet-triplet splitting via the missing partner mechanism. With models involving $144+\overline{144}$ and $560+\overline{560}$ of Higgs fields the analysis of textures is rather different from the conventional approach (see, e.g., Ref. [155]). Here the couplings of the 144 or of 560 with matter can over only occur at the quartic level which are suppressed by the Planck scale. While such couplings can produce the right size of Yukawas for the first and the second generation fermions, one requires additional matter fields to generate the right size of third generation masses. In the analysis using $144+\overline{144}$ of Higgs it is found [149, 156] that $b-t-\tau$ unification does not necessarily require a large tan $\beta$ [157].

We now briefly comment on the current status of proton stability. Proton decay is an important discriminant for models based on GUTs and strings [158. In SUSY GUTs and in strings proton decay can proceed under the constraints of $\mathrm{R}$ parity via baryon and lepton number violating dimension five and dimension six operators. For dimension six operators the dominant decay mode of the proton is $p \rightarrow e^{+} \pi^{0}$ which proceeds via leptoquark exchange

(for reviews see Refs. [159 161]) so that $\Gamma\left(p \rightarrow e^{+} \pi^{0}\right) \simeq \alpha_{G}^{2} \frac{m_{p}^{5}}{M_{V}^{4}}$ which gives $\tau\left(p \rightarrow e^{+} \pi^{0}\right) \simeq 10^{36 \pm 1} y r s$. This mode may allow one to discriminate between GUTs and strings. Thus generic D brane models allow only $10^{2} \overline{1} 0^{2} \mathrm{SU}(5)$ type dimension six operators which give $p \rightarrow \pi^{0} e_{L}^{+}$while SU(5) GUTs also have $10105 \overline{5}$ which allow $p \rightarrow \pi^{0} e_{R}^{+}[162$. Additionally the $S U(5)$ GUT models also give the decay $p \rightarrow \pi^{+} \nu$ which is not allowed in generic D brane models. However, there exist special regions of intersecting D brane models where the operator $10 \overline{0} \overline{5}$ is indeed allowed and the purely stringy proton decay rate can be of the same order as the one from SU(5) GUTs including the mode $p \rightarrow \pi^{+} \nu$ [163. The current status of proton decay via baryon and lepton number violating dimension six operators is as follows: Superkamiokande gives the limit [164] $\tau\left(p \rightarrow e^{+} \pi^{0}\right)>1.4 \times 10^{34}$ yrs while in the future Hyper-K is expected to achieve a sensitivity [164 of $\tau\left(p \rightarrow e^{+} \pi^{0}\right)>1 \times 10^{35}$ yrs. Regarding the proton decay arising from baryon and lepton number violating dimension five operators via the Higgsino triplet exchange the dominant mode is $\bar{\nu} K^{+}$which depends sensitively on the sparticle spectrum. Proton decay also has a significant dependence on CP phases [165. The current experimental limit from Super-K is $\tau\left(p \rightarrow \bar{\nu} K^{+}\right)>4 \times 10^{33}$ yrs, while in the future it is expected that Hyper-K may reach a sensitivity of $\tau\left(p \rightarrow \bar{\nu} K^{+}\right)>2 \times 10^{34}$ yrs. Proton decay from dimension five operators in GUTs and strings in typically too rapid if the sparticle spectrum is light and one needs a cancellation mechanism or other means to stabilize the proton [159]. Proton stability from these operators is more easily achieved when $m_{0}$ is large and REWSB occurs on the Hyperbolic Branch [74].

\section{A. Radiative Breaking and R Parity}

As discussed above one needs R parity for SUSY dark matter and in GUT models, for proton stability. But R Parity even if preserved at the GUT scale can be broken by RG effects [166] (for early works on R parity see Refs. [167, 168]). Thus suppose $\mathrm{R}$ parity arises from a $B-L$ gauge symmetry, i.e., $R=(-1)^{2 s+3(B-L)}$. After spontaneous breaking $\mathrm{R}$ parity will be preserved if $3(B-L)$ is an even integer. However, even if $R$ parity is preserved at the GUT scale, 
it can be broken by RG effects. Thus consider an extension of MSSM with a $U(1)_{B-L}$ symmetry. Here for anomaly cancellation one needs three right handed neutrino fields $\nu^{c}$. The extended superpotential in this case is

$$
W=W_{M S S M}+h_{\nu} L H \nu^{c}+h_{\nu^{c}} \nu^{c} \nu^{c} \Phi+\mu_{\Phi} \Phi \bar{\Phi} .
$$

The $B-L$ quantum numbers of the new fields $\left(\nu^{c}, \Phi, \bar{\Phi}\right)$ are $(1,-2,2)$. A VEV growth for $\nu^{c}$ will break $R$ parity, but a VEV growth for $\Phi$ won't. However, the beta functions due to the coupling of the $\Phi$ to the $\nu^{c}$ can turn the mass of $\tilde{\nu}^{c}$ tachyonic which leads to a VEV growth for $\tilde{\nu}^{c}$ and a violation of $R$ parity. A Stueckelberg mass growth for the $B-L$ gauge boson (see Sec.(VI) for a review of the Stueckelberg mechanism) will preserve $R$ parity since there no $\Phi, \bar{\Phi}$ fields are needed to break the symmetry 169 .

\section{STUECKELBERG EXTENSIONS}

In the area of string phenomenology one may classify the current work broadly in the following areas: (i) String model building which includes looking for string vacua which have the standard model gauge group, have three generations of quarks and leptons, solve the doublet-triplet problem, and have no exotics. Desirable models should also have a mechanism for generating soft terms and a mechanism for understanding the smallness of the cosmological constant; (ii) Investigation of model independent signatures in low scale compactifications, e.g., via scattering amplitudes; (iii) Investigation of other generic or model independent features: This includes investigation of hidden sectors which are endemic in string models. Here there is considerable work on the investigations of models where communication between the visible sector and the hidden sector occurs either via gauge kinetic energy mixing or from mixings via the Stueckelberg mechanism. We will focus on the last item, i.e., the implications of the hidden sectors in string theory for particle physics. Hidden sectors first arose in gravity mediated breaking of supersymmetry where supersymmetry is broken in a hidden sector and communicated to the visible sector by gravitational interactions. However, the hidden sector may play a larger role. We illustrate this by an example where the communication between the hidden sector and the visible sector takes place not by gravity but by a gauge field via the Stueckelberg mechanism. Now the Stueckelberg mechanism is quite generic in string compactifications and in the reduction of the higher dimensional theories to four dimensions. As an example consider terms in the Lagrangian of the form

$$
L_{1}=-\frac{1}{12} H^{\mu \nu \lambda} H_{\mu \nu \lambda}+\frac{m}{4} \epsilon^{\mu \nu \rho \sigma} F_{\mu \nu} B_{\rho \sigma}
$$

where $F_{\mu \nu}$ is the field strength of a $U(1)$ gauge field $A_{\mu}$ and $B_{\mu \nu}$ is anti-symmetric one form field, and $H^{\mu \nu \lambda}$ is the corresponding field strength given by $H_{\mu \nu \rho}=\partial_{\mu} B_{\nu \rho}+\partial_{\nu} B_{\rho \mu}+\partial_{\rho} B_{\mu \nu}$. A simple set of field redefinitions reduce $L_{1}$ to the form

$$
L_{2}=-\frac{m^{2}}{2}\left(A_{\sigma}+\partial_{\sigma} \sigma\right)^{2} .
$$

where $\sigma$ is related to $H^{\mu \nu \rho}$ so that $H^{\mu \nu \rho}=-m \epsilon^{\mu \nu \rho \sigma}\left(A_{\sigma}+\partial_{\sigma} \sigma\right)$.

Eq. 22 is a very simple form of terms that arise in string theory. Thus one finds that the Stueckelberg mechanism arises quite naturally in strings. Let us assume now that one has a hidden sector endowed with a $U(1)_{X}$ gauge group under which the standard model particles are all neutral. Normally there would be no communication between the standard model sector and the hidden sector in this case. Suppose, however, there was a small mixing between the hidden and the visible sector via either the kinetic energy sector or via the Stueckelberg sector. Thus suppose we have a mixing between the hypercharge field $B_{\mu}$ and the $U(1)_{X}$ gauge field $C_{\mu}$ so that the Stueckelberg interaction takes the form [143, 170.

$$
L_{S t}=-\frac{1}{2}\left(\partial_{\mu} \sigma+M_{1} C_{\mu}+M_{2} B_{\mu}\right)^{2}
$$

Combining it with the Higgs mechanism one finds that the vector boson sector now becomes a $3 \times 3$ matrix rather than a $2 \times 2$ matrix as in the case of the standard model and for the MSSM [141, 143, 144, 170,172. As a consequence of Eq. (24) the gauge boson of the hidden sector will couple with the quarks and the lepton of the visible sector and the coupling will be determined by the ratio $\epsilon=M_{2} / M_{1}$ and such a $Z^{\prime}$ can be constrained by experimental data (for other related works see, e.g., [173, 174]). The current constraints on $\epsilon$ arising from the D $\varnothing$ Collaboration [175] and the CMS detector Collaboration [176] are exhibited in Fig. 7]. The analysis of D $\varnothing$ Collaboration [175] (left panel) was based on $5.4 \mathrm{fb}^{-1}$ of data and shows that the $Z_{\mathrm{St}}^{\prime}$ mass could be as low as $\sim 200 \mathrm{GeV}$ for $\epsilon=0.06$. The analysis

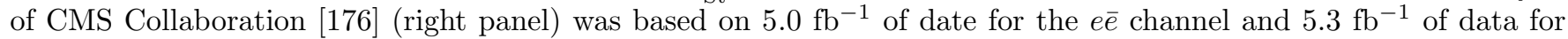
the $\mu^{+} \mu^{-}$channel. Here one finds that the $Z_{\text {St }}^{\prime}$ can be as low as $\sim 600 \mathrm{GeV}$ at $95 \%$ CL for the case $\epsilon=0.06$. 

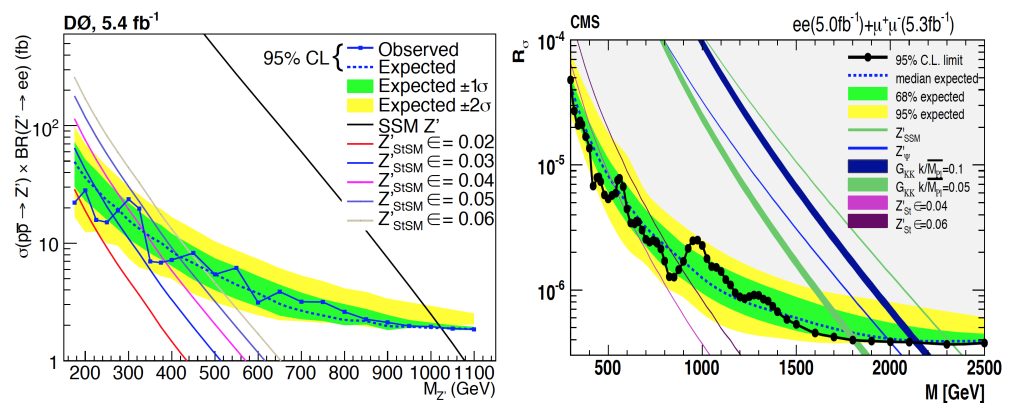

FIG. 7: Left panel: The constraint on the $Z_{\text {St }}^{\prime}$ mass vs the mixing parameter $\epsilon$ in the Stueckelberg extension of the standard model and of the minimal supersymmetric standard model by the D $\varnothing$ Collaboration with $5.4 \mathrm{fb}^{-1}$ of data at the Tevatron (Taken from Ref. 175). Right panel: The constraint on the $Z_{\mathrm{St}}^{\prime}$ mass vs $\epsilon$ by the CMS Collaboration using $5.0 \mathrm{fb}^{-1}$ of data for the $e \bar{e}$ mode and $5.3 \mathrm{fb}^{-1}$ of data for the $\mu \bar{\mu}$ mode (Taken from Ref. [176]). One finds that a Stueckelberg $Z_{\mathrm{St}}^{\prime}$ as low as a few hundred $\mathrm{GeV}$ is still possible.
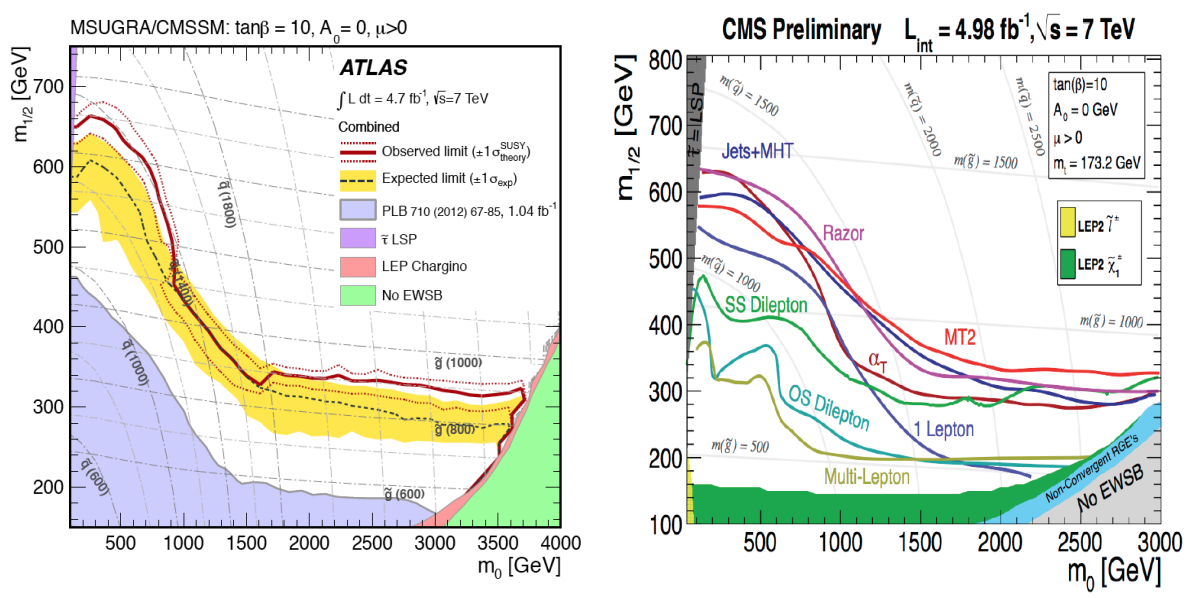

FIG. 8: The left panel: The reach plot in the $m_{1 / 2}-m_{0}$ plane by the ATLAS Collaboration with $4.7 \mathrm{fb}^{-1} \mathrm{of}^{\mathrm{integrated}}$ luminosity (taken from Ref. [177]). Right panel: The reach plot in the $m_{1 / 2}-m_{0}$ plane by the CMS Collaboration at $\sqrt{s}=7$ $\mathrm{TeV}$ with $4.98 \mathrm{fb}^{-1}$ of integrated luminosity (taken from Campagnari's talk at SUSY2012 [178]).

\section{PROSPECTS FOR SUSY}

If one believes that the Higgs boson is elementary then unless one is willing to live with a large fine tuning, supersymmetry is the clear alternative. However, the $\sim 125 \mathrm{GeV}$ Higgs mass puts a severe constraint on supersymmetric models. This is because a large loop correction which lifts the tree level Higgs mass of below $M_{Z}$ to $\sim 125 \mathrm{GeV}$ is needed and not every model can support such a large correction. Consequently several models get eliminated while others are severely constrained [12]. The mSUGRA model as well as the non-universal SUGRA models can lift the tree level Higgs mass to what is observed. Indeed in mSUGRA the Higgs boson mass is predicted to lie below $\sim 130$ $\mathrm{GeV}$ and it is interesting that the experimental value ended up below this limit [11, 12, 14. The way one generates a large correction to the Higgs boson mass is to have a large $m_{0}$ which tends to make the first two generation sfermions heavy. However, it turns out that in this high scale model there exist very significant regions of the parameter space where $\mu$ is small, i.e., as low as $\sim 200 \mathrm{GeV}$ as well as the gluino, charginos and neutralinos can be light essentially limited by the experimental lower limits. Thus the most likely candidates for discovery at the LHC are the gluino, the chargino, the neutralino and some of the third generation sfermions. The observation of the Higgs boson provides a strong argument to proceed directly to $\sqrt{s}=14 \mathrm{TeV}$ to discover SUSY. In summary the discovery of the Higgs boson is good news for supersymmetry. A full fledged search for supersymmetry is now desirable. The current exclusion plots in the $m_{0}-m_{1 / 2}$ plane by the ATLAS and CMS Collaborations are given in Fig. (8). A recent analysis for the reach of LHC at $\sqrt{s}=14 \mathrm{TeV}$ is given in Ref. 179. and the analysis shows that the discovery reach at the LHC at $\sqrt{s}=14 \mathrm{TeV}$ with an integrated luminosity up to $3000 \mathrm{fb}^{-1}$ will be extended by significant amounts. 


\section{THE WAY FORWARD}

After the historic results from CERN this year, there are clearly two parallel paths that need to be pursued: the first relates to determining more accurately the properties of the discovered boson and the second to a renewed effort to discover supersymmetry. Regarding the newly discovered boson the way forward is clear. Although there is little doubt that the newly discovered boson is the long sought after Higgs boson, one must fully establish its spin and CP properties. Second, one needs to measure accurately the couplings of the new boson with quarks, leptons and vector bosons. An identifiable deviation from the standard model Higgs prediction would be a guide to the nature of new physics beyond the standard model. However, accurate measurement of the Higgs boson couplings are difficult and as mentioned earlier such measurements at the LHC at best could be at the 10-20\% level by some estimates [114]. In view of that it is timely to start thinking about Higgs factories, i.e., machines beyond the LHC era, where such tasks could be better accomplished. Aside from ILC or CLIC, other possibilities discussed are the muon collider 180 and more recently a $\gamma \gamma$ Higgs factory SAPPHiRE (Small Accelerator for Photon-Photon Higgs production using Recirculating Electrons) [181. Aside from the accurate measurement of the properties of the new boson, the main focus now ought to shift to the discovery of supersymmetry.

As mentioned already if one believes in the elementarity of the Higgs boson then supersymmetry is the most desirable solution. The analysis including the constraint that the Higgs boson mass be $\sim 125 \mathrm{GeV}$, requires that in SUGRA model with universal boundary conditions, the scalar mass $m_{0}$, is large which makes the first two generations of sfermions heavy, i.e., of size several TeV. However, some of the third generation sfermions can be light, specifically, the stop and the stau, due to large trilinear couplings. Additionally the gauginos, i.e., the gluino, the chargino, and the neutralino can be light and these could be detected at higher energy and with increased luminosity. It is also possible that the light sparticles may have been missed in LHC searches for a variety of reasons. Thus, for example, if the mass gap between the LSP and the other low lying sparticles is small, the decay products of sparticles may not pass the conventional LHC cuts allowing SUSY to remain undetected. Alternately without R parity the conventional missing energy signals would not apply. Thus, more comprehensive signature analysis are called for exhausting the allowed possibilities in the sparticle landscape $[182,183$. Thus the possibility that several sparticles can be relatively light consistent with a $\sim 125 \mathrm{GeV}$ Higgs mass is promising for SUSY discovery with further data at the LHC.

\section{Acknowledgments}

This work is supported in part by the U.S. National Science Foundation (NSF) grants PHY-0757959 and PHY070467 and DOE NERSC grant DE-AC02-05CH11231.

[1] F. Englert and R. Brout, Phys. Rev. Lett. 13, 321 (1964).

[2] P. W. Higgs, Phys. Rev. Lett. 13, 508 (1964).

[3] G. Guralnik, C. Hagen and T. Kibble, Phys. Rev. Lett. 13, 585 (1964).

[4] S. Weinberg, Phys.Rev.Lett. 19, 1264 (1967).

[5] A. Salam, Elementary paricle theory (Almqvist and Wiksells, Stockholm, 1968), p. 367.

[6] ATLAS Collaboration Collaboration (G. Aad et al.), Phys.Lett. B716, 1 (2012), arXiv:1207.7214 [hep-ex]

[7] CMS Collaboration Collaboration (S. Chatrchyan et al.), Phys.Lett. B716, 30 (2012), arXiv:1207.7235 [hep-ex].

[8] CMS Collaboration Collaboration (V. Khachatryan et al.), Phys.Lett. B698, 196 (2011), arXiv:1101.1628 [hep-ex].

[9] Atlas Collaboration Collaboration (G. Aad et al.), Phys.Rev.Lett. 106, 131802 (2011), arXiv:1102.2357 [hep-ex]

[10] A. H. Chamseddine, R. L. Arnowitt and P. Nath, Phys. Rev. Lett. 49, 970 (1982).

[11] S. Akula, B. Altunkaynak, D. Feldman, P. Nath and G. Peim, Phys. Rev. D85, 075001 (2012), arXiv:1112.3645 [hep-ph].

[12] A. Arbey, M. Battaglia, A. Djouadi and F. Mahmoudi (2012), arXiv:1207.1348 [hep-ph]

[13] J. Ellis and K. A. Olive, Eur.Phys.J. C72, 2005 (2012), arXiv:1202.3262 [hep-ph]

[14] S. Akula, P. Nath and G. Peim (2012), arXiv:1207.1839 [hep-ph]

[15] L. Maiani, A. Polosa and V. Riquer (2012), arXiv:1209.4816 [hep-ph]

[16] C.-N. Yang, Phys.Rev. 77, 242 (1950).

[17] S. Choi, . Miller, D.J., M. Muhlleitner and P. Zerwas, Phys.Lett. B553, 61 (2003), arXiv:hep-ph/0210077 [hep-ph]

[18] J. Ellis and D. S. Hwang (2012), arXiv:1202.6660 [hep-ph]

[19] G. S. Guralnik, Int.J.Mod.Phys. A24, 2601 (2009), arXiv:0907.3466 [physics.hist-ph]. 
[20] Tevatron New Physics Higgs Working Group, CDF Collaboration, D0 Collaboration Collaboration (C. Group, D. Collaborations, the Tevatron New Physics and H. Working) (2012), arXiv:1207.0449 [hep-ex].

[21] J. F. Gunion, H. E. Haber, G. L. Kane and S. Dawson, Front.Phys. 80, 1 (2000).

[22] M. Carena, S. Gori, N. R. Shah and C. E. Wagner, JHEP 1203, 014 (2012), arXiv:1112.3336 [hep-ph].

[23] M. Carena, I. Low and C. E. Wagner, JHEP 1208, 060 (2012), arXiv:1206.1082 [hep-ph].

[24] G. F. Giudice, P. Paradisi and A. Strumia (2012), arXiv:1207.6393 [hep-ph].

[25] A. Joglekar, P. Schwaller and C. E. Wagner (2012), arXiv:1207.4235 [hep-ph]

[26] N. Arkani-Hamed, K. Blum, R. T. D'Agnolo and J. Fan (2012), arXiv:1207.4482 [hep-ph]

[27] H. An, T. Liu and L.-T. Wang (2012), arXiv:1207.2473 [hep-ph].

[28] T. Abe, N. Chen and H.-J. He (2012), arXiv:1207.4103 [hep-ph]

[29] J. Chang, K. Cheung, P.-Y. Tseng and T.-C. Yuan (2012), arXiv:1206.5853 [hep-ph]

[30] J. Baglio, A. Djouadi and R. Godbole, Phys.Lett. B716, 203 (2012), arXiv:1207.1451 [hep-ph].

[31] D. Dicus and V. Mathur, Phys.Rev. D7, 3111 (1973).

[32] B. W. Lee, C. Quigg and H. Thacker, Phys.Rev.Lett. 38, 883 (1977).

[33] G. Degrassi, S. Di Vita, J. Elias-Miro, J. R. Espinosa, G. F. Giudice et al., JHEP 1208, 098 (2012), arXiv:1205.6497 [hep-ph].

[34] S. Alekhin, A. Djouadi and S. Moch, Phys.Lett. B716, 214 (2012), arXiv:1207.0980 [hep-ph].

[35] I. Masina (2012), arXiv:1209.0393 [hep-ph].

[36] A. H. Chamseddine and A. Connes, JHEP 1209, 104 (2012), arXiv:1208.1030 [hep-ph].

[37] L. A. Anchordoqui, I. Antoniadis, H. Goldberg, X. Huang, D. Lust et al. (2012), arXiv:1208.2821 [hep-ph]

[38] P. Nath, R. L. Arnowitt and A. H. Chamseddine, Applied $N=1$ Supergravity, ICTP Series in Theoretical Physics, Vol. 1 (World Scientific, Singapore, 1984).

[39] M. S. Carena and H. E. Haber, Prog.Part.Nucl.Phys. 50, 63 (2003), arXiv:hep-ph/0208209 [hep-ph]

[40] A. Djouadi, Phys.Rept. 459, 1 (2008), arXiv:hep-ph/0503173 [hep-ph]

[41] P. Nath and R. L. Arnowitt, Phys.Lett. B56, 177 (1975).

[42] R. L. Arnowitt, P. Nath and B. Zumino, Phys.Lett. B56, 81 (1975).

[43] D. Z. Freedman, P. van Nieuwenhuizen and S. Ferrara, Phys.Rev. D13, 3214 (1976).

[44] S. Deser and B. Zumino, Phys.Lett. B62, 335 (1976).

[45] P. Nath and R. L. Arnowitt, Phys.Lett. B65, 73 (1976).

[46] Y. Sumitomo and S.-H. H. Tye, JCAP 1208, 032 (2012), arXiv:1204.5177 [hep-th].

[47] B. S. Acharya, K. Bobkov, G. L. Kane, J. Shao and P. Kumar, Phys.Rev. D78, 065038 (2008), arXiv:0801.0478 [hep-ph].

[48] J. P. Conlon, F. Quevedo and K. Suruliz, JHEP 0508, 007 (2005), arXiv:hep-th/0505076 [hep-th]

[49] P. Nath, R. L. Arnowitt and A. H. Chamseddine, Nucl. Phys. B227, 121 (1983).

[50] L. J. Hall, J. D. Lykken and S. Weinberg, Phys.Rev. D27, 2359 (1983).

[51] P. Nath, 3 (2003), arXiv:hep-ph/0307123 [hep-ph].

[52] G. L. Kane, C. F. Kolda and J. D. Wells, Phys.Rev.Lett. 70, 2686 (1993), arXiv:hep-ph/9210242 [hep-ph]

[53] J. Cao, Z. Heng, J. M. Yang and J. Zhu (2012), arXiv:1207.3698 [hep-ph].

[54] A. G. Cohen and M. Schmaltz (2012), arXiv:1207.3495 [hep-ph]

[55] T. Cheng, J. Li, T. Li, X. Wan, Y. k. Wang et al. (2012), arXiv:1207.6392 [hep-ph]

[56] P. B. Dev, S. Mondal, B. Mukhopadhyaya and S. Roy (2012), arXiv:1207.6542 [hep-ph]

[57] M. A. Ajaib, I. Gogoladze and Q. Shafi (2012), arXiv:1207.7068 [hep-ph].

[58] MAP Collaboration Collaboration (arXiv:1207.7068 [hep-ph]) (2012).

[59] N. Liu, L. Wu, P. W. Wu and J. M. Yang (2012), arXiv:1208.3413 [hep-ph].

[60] L. E. Ibanez, F. Marchesano, D. Regalado and I. Valenzuela, JHEP 1207, 195 (2012), arXiv:1206.2655 [hep-ph].

[61] A. Hebecker, A. K. Knochel and T. Weigand, JHEP 1206, 093 (2012), arXiv:1204.2551 [hep-th]

[62] L. Aparicio, D. Cerdeno and L. Ibanez, JHEP 1204, 126 (2012), arXiv:1202.0822 [hep-ph]

[63] T. Li, J. A. Maxin, D. V. Nanopoulos and J. W. Walker (2012), arXiv:1208.1999 [hep-ph].

[64] C. Boehm, J. Da Silva, A. Mazumdar and E. Pukartas (2012), arXiv:1205.2815 [hep-ph].

[65] J. Ellis, D. S. Hwang, V. Sanz and T. You (2012), arXiv:1208.6002 [hep-ph].

[66] M. W. Cahill-Rowley, J. L. Hewett, A. Ismail and T. G. Rizzo (2012), arXiv:1206.5800 [hep-ph].

[67] J. L. Feng and D. Sanford (2012), arXiv:1205.2372 [hep-ph]

[68] P. F. Perez and S. Spinner (2012), arXiv:1209.5769 [hep-ph]

[69] M. Dhuria and A. Misra (2012), arXiv:1207.2774 [hep-ph]

[70] C. Wymant (2012), arXiv:1208.1737 [hep-ph].

[71] A. Arbey, M. Battaglia, A. Djouadi, F. Mahmoudi and J. Quevillon, Phys.Lett. B708, 162 (2012), arXiv:1112.3028 [hep-ph]

[72] S. Akula, M. Liu, P. Nath and G. Peim, Phys. Lett. B709, 192 (2012), arXiv:1111.4589 [hep-ph].

[73] R. L. Arnowitt and P. Nath, Phys.Rev. D46, 3981 (1992).

[74] K. L. Chan, U. Chattopadhyay and P. Nath, Phys.Rev. D58, 096004 (1998), arXiv:hep-ph/9710473 [hep-ph]

[75] U. Chattopadhyay, A. Corsetti and P. Nath, Phys.Rev. D68, 035005 (2003), arXiv:hep-ph/0303201 [hep-ph]

[76] H. Baer, V. Barger, P. Huang and X. Tata, JHEP 1205, 109 (2012), arXiv:1203.5539 [hep-ph].

[77] J. L. Feng, K. T. Matchev and T. Moroi, Phys.Rev.Lett. 84, 2322 (2000), arXiv:hep-ph/9908309 [hep-ph].

[78] D. Feldman, G. Kane, E. Kuflik and R. Lu, Phys.Lett. B704, 56 (2011), arXiv:1105.3765 [hep-ph]. 
[79] R. Barbieri and G. Giudice, Nucl.Phys. B306, 63 (1988).

[80] M. Drees, Int.J.Mod.Phys. A4, 3635 (1989).

[81] U. Ellwanger, C. Hugonie and A. M. Teixeira, Phys.Rept. 496, 1 (2010), arXiv:0910.1785 [hep-ph]

[82] P. Batra, A. Delgado, D. E. Kaplan and T. M. Tait, JHEP 0402, 043 (2004), arXiv:hep-ph/0309149 [hep-ph].

[83] A. Maloney, A. Pierce and J. G. Wacker, JHEP 0606, 034 (2006), arXiv:hep-ph/0409127 [hep-ph]

[84] L. J. Hall, D. Pinner and J. T. Ruderman, JHEP 1204, 131 (2012), arXiv:1112.2703 [hep-ph]

[85] H. P. Nilles, M. Srednicki and D. Wyler, Phys.Lett. B124, 337 (1983).

[86] J. Bagger and E. Poppitz, Phys.Rev.Lett. 71, 2380 (1993), arXiv:hep-ph/9307317 [hep-ph].

[87] L. Randall and M. Reece (2012), arXiv:1206.6540 [hep-ph]

[88] C. Brust, A. Katz and R. Sundrum, JHEP 1208, 059 (2012), arXiv:1206.2353 [hep-ph]

[89] R. Essig, E. Izaguirre, J. Kaplan and J. G. Wacker, JHEP 1201, 074 (2012), arXiv:1110.6443 [hep-ph]

[90] B. Allanach and B. Gripaios, JHEP 1205, 062 (2012), arXiv:1202.6616 [hep-ph]

[91] ATLAS Collaboration Collaboration (G. Aad et al.) (2012), arXiv:1208.1447 [hep-ex].

[92] P. Nath and R. L. Arnowitt, Phys. Rev. D56, 2820 (1997), arXiv:hep-ph/9701301 [hep-ph]

[93] H. Baer, V. Barger, P. Huang, A. Mustafayev and X. Tata (2012), arXiv:1207.3343 [hep-ph]

[94] A. Corsetti and P. Nath, Phys.Rev. D64, 125010 (2001), arXiv:hep-ph/0003186 [hep-ph].

[95] J. Jaeckel and V. V. Khoze (2012), arXiv:1205.7091 [hep-ph]

[96] H. E. Haber (1994), arXiv:hep-ph/9501320 [hep-ph]

[97] R. S. Gupta, H. Rzehak and J. D. Wells (2012), arXiv:1206.3560 [hep-ph]

[98] F. Feroz, M. Hobson and M. Bridges, Mon. Not. Roy. Astron. Soc. 398, 1601 (2009), arXiv: 0809.3437 [astro-ph]

[99] R. Trotta, F. Feroz, M. P. Hobson, L. Roszkowski and R. Ruiz de Austri, JHEP 0812, 024 (2008), arXiv:0809.3792 [hep-ph].

[100] S. R. Choudhury and N. Gaur, Phys.Lett. B451, 86 (1999), arXiv:hep-ph/9810307 [hep-ph].

[101] K. Babu and C. F. Kolda, Phys.Rev.Lett. 84, 228 (2000), arXiv:hep-ph/9909476 [hep-ph]

[102] C. Bobeth, T. Ewerth, F. Kruger and J. Urban, Phys.Rev. D64, 074014 (2001), arXiv:hep-ph/0104284 [hep-ph].

[103] A. Dedes, H. K. Dreiner and U. Nierste, Phys.Rev.Lett. 87, 251804 (2001), arXiv:hep-ph/0108037 [hep-ph]

[104] R. L. Arnowitt, B. Dutta, T. Kamon and M. Tanaka, Phys.Lett. B538, 121 (2002), arXiv:hep-ph/0203069 [hep-ph]

[105] J. Mizukoshi, X. Tata and Y. Wang, Phys.Rev. D66, 115003 (2002), arXiv:hep-ph/0208078 [hep-ph]

[106] T. Ibrahim and P. Nath, Phys.Rev. D67, 016005 (2003), arXiv:hep-ph/0208142 [hep-ph].

[107] D. Feldman, Z. Liu and P. Nath, Phys.Rev. D81, 117701 (2010), arXiv:1003.0437 [hep-ph]

[108] N. D. Christensen, T. Han and T. Li (2012), arXiv:1206.5816 [hep-ph]

[109] M. Klute, R. Lafaye, T. Plehn, M. Rauch and D. Zerwas (2012), arXiv:1205.2699 [hep-ph]

[110] R. Lafaye, T. Plehn, M. Rauch, D. Zerwas and M. Duhrssen, JHEP 0908, 009 (2009), arXiv:0904.3866 [hep-ph].

[111] M. Duhrssen, S. Heinemeyer, H. Logan, D. Rainwater, G. Weiglein et al., Phys.Rev. D70, 113009 (2004), arXiv: hep-ph/0406323 [hep-ph].

[112] D. Zeppenfeld, R. Kinnunen, A. Nikitenko and E. Richter-Was, Phys.Rev. D62, 013009 (2000), arXiv:hep-ph/0002036 [hep-ph].

[113] T. Plehn and M. Rauch (2012), arXiv:1207.6108 [hep-ph].

[114] M. E. Peskin (2012), arXiv:1207.2516 [hep-ph]

[115] T. Yuan, R. L. Arnowitt, A. H. Chamseddine and P. Nath, Z.Phys. C26, 407 (1984).

[116] D. A. Kosower, L. M. Krauss and N. Sakai, Phys.Lett. B133, 305 (1983).

[117] A. Hoecker, Nucl.Phys.Proc.Suppl. 218, 189 (2011), arXiv:1012.0055 [hep-ph].

[118] K. Hagiwara, R. Liao, A. D. Martin, D. Nomura and T. Teubner, J.Phys.G G38, 085003 (2011), arXiv:1105.3149 [hep-ph].

[119] T. Aoyama, M. Hayakawa, T. Kinoshita and M. Nio (2012), arXiv:1205.5370 [hep-ph].

[120] S. Heinemeyer, D. Stockinger and G. Weiglein, Nucl.Phys. B699, 103 (2004), arXiv:hep-ph/0405255 [hep-ph]

[121] T. Moroi and Y. Okada, Phys.Lett. B295, 73 (1992).

[122] K. Babu, I. Gogoladze, M. U. Rehman and Q. Shafi, Phys.Rev. D78, 055017 (2008), arXiv:0807.3055 [hep-ph]

[123] S. P. Martin, Phys.Rev. D82, 055019 (2010), arXiv:1006.4186 [hep-ph]

[124] S. P. Martin, Phys.Rev. D81, 035004 (2010), arXiv:0910.2732 [hep-ph]

[125] T. Moroi, R. Sato and T. T. Yanagida, Phys.Lett. B709, 218 (2012), arXiv:1112.3142 [hep-ph]

[126] M. Endo, K. Hamaguchi, S. Iwamoto and N. Yokozaki, Phys.Rev. D84, 075017 (2011), arXiv:1108.3071 [hep-ph].

[127] M. Endo, K. Hamaguchi, S. Iwamoto, K. Nakayama and N. Yokozaki, Phys.Rev. D85, 095006 (2012), arXiv:1112.6412 [hep-ph].

[128] S. Akula et al. (2012), in preparation.

[129] XENON100 Collaboration (E. Aprile et al.) (2012), arXiv:1207.5988 [astro-ph.C0]

[130] XENON100 Collaboration (E. Aprile et al.), Phys. Rev. Lett. 107, 131302 (2011), arXiv:1104.2549 [astro-ph.C0]

[131] XENON100 Collaboration (E. Aprile et al.), Phys. Rev. D84, 052003 (2011), arXiv:1103.0303 [hep-ex].

[132] XENON100 Collaboration (E. Aprile et al.), Phys. Rev. Lett. 105, 131302 (2010), arXiv:1005.0380 [astro-ph.C0]

[133] XENON1T collaboration Collaboration (E. Aprile) (2012), arXiv:1206.6288 [astro-ph.IM].

[134] SuperCDMS Collaboration, B. Cabrera, Supercdms development project (April 2005), Fermilab Program Advisory Committee.

[135] R. L. Arnowitt and P. Nath, Phys. Rev. Lett. 69, 725 (1992).

[136] WMAP Collaboration Collaboration (E. Komatsu et al.), Astrophys.J.Suppl. 192, 18 (2011), arXiv:1001.4538 
[astro-ph.CO]

[137] D. E. Kaplan, M. A. Luty and K. M. Zurek, Phys.Rev. D79, 115016 (2009), arXiv:0901.4117 [hep-ph].

[138] H. Davoudiasl and R. N. Mohapatra (2012), arXiv:1203.1247 [hep-ph].

[139] J. A. Harvey and M. S. Turner, Phys.Rev. D42, 3344 (1990).

[140] E. W. Kolb and M. S. Turner (1989), Addison-Wesley, Pub. Company

[141] B. Kors and P. Nath, Phys.Lett. B586, 366 (2004), arXiv:hep-ph/0402047 [hep-ph]

[142] B. Kors and P. Nath, JHEP 0412, 005 (2004), arXiv:hep-ph/0406167 [hep-ph]

[143] B. Kors and P. Nath, JHEP 0507, 069 (2005), arXiv:hep-ph/0503208 [hep-ph]

[144] D. Feldman, Z. Liu and P. Nath, JHEP 0611, 007 (2006), arXiv: hep-ph/0606294 [hep-ph].

[145] D. Feldman, Z. Liu and P. Nath, Phys.Rev. D75, 115001 (2007), arXiv:hep-ph/0702123 [HEP-PH].

[146] W.-Z. Feng, P. Nath and G. Peim, Phys.Rev. D85, 115016 (2012), arXiv:1204.5752 [hep-ph]

[147] K. R. Dienes and B. Thomas, Phys.Rev. D85, 083523 (2012), arXiv:1106.4546 [hep-ph]

[148] K. R. Dienes, S. Su and B. Thomas (2012), arXiv:1204.4183 [hep-ph]

[149] K. Babu, I. Gogoladze, P. Nath and R. M. Syed, Phys.Rev. D74, 075004 (2006), arXiv:hep-ph/0607244 [hep-ph]

[150] K. Babu, I. Gogoladze, P. Nath and R. M. Syed, Phys.Rev. D72, 095011 (2005), arXiv:hep-ph/0506312 [hep-ph]

[151] B. Grinstein, Nucl.Phys. B206, 387 (1982).

[152] A. Masiero, D. V. Nanopoulos, K. Tamvakis and T. Yanagida, Phys.Lett. B115, 380 (1982).

[153] K. Babu, I. Gogoladze and Z. Tavartkiladze, Phys.Lett. B650, 49 (2007), arXiv:hep-ph/0612315 [hep-ph].

[154] K. Babu, I. Gogoladze, P. Nath and R. M. Syed, Phys.Rev. D85, 075002 (2012), arXiv:1112.5387 [hep-ph]

[155] U. Chattopadhyay and P. Nath, Phys.Rev. D65, 075009 (2002), arXiv:hep-ph/0110341 [hep-ph]

[156] P. Nath and R. M. Syed, Phys.Rev. D81, 037701 (2010), arXiv:0909.2380 [hep-ph]

[157] B. Ananthanarayan, G. Lazarides and Q. Shafi, Phys.Rev. D44, 1613 (1991).

[158] R. L. Arnowitt and P. Nath, Phys.Rev. D49, 1479 (1994), arXiv:hep-ph/9309252 [hep-ph]

[159] P. Nath and P. Fileviez Perez, Phys.Rept. 441, 191 (2007), arXiv:hep-ph/0601023 [hep-ph]

[160] S. Raby, T. Walker, K. Babu, H. Baer, A. Balantekin et al. (2008), arXiv:0810.4551 [hep-ph].

[161] J. Hewett, H. Weerts, R. Brock, J. Butler, B. Casey et al. (2012), arXiv:1205.2671 [hep-ex].

[162] I. R. Klebanov and E. Witten, Nucl.Phys. B664, 3 (2003), arXiv:hep-th/0304079 [hep-th].

[163] M. Cvetic and R. Richter, Nucl.Phys. B762, 112 (2007), arXiv:hep-th/0606001 [hep-th]

[164] K. Abe, T. Abe, H. Aihara, Y. Fukuda, Y. Hayato et al. (2011), arXiv:1109.3262 [hep-ex].

[165] T. Ibrahim and P. Nath, Rev.Mod.Phys. 80, 577 (2008), arXiv:0705.2008 [hep-ph].

[166] V. Barger, P. Fileviez Perez and S. Spinner, Phys.Rev.Lett. 102, 181802 (2009), arXiv:0812.3661 [hep-ph]

[167] C. Aulakh and R. N. Mohapatra, Phys.Lett. B119, 136 (1982).

[168] S. Khalil and A. Masiero, Phys.Lett. B665, 374 (2008), arXiv:0710.3525 [hep-ph]

[169] D. Feldman, P. Fileviez Perez and P. Nath, JHEP 1201, 038 (2012), arXiv:1109.2901 [hep-ph]

[170] B. Kors and P. Nath, 437 (2004), arXiv:hep-ph/0411406 [hep-ph]

[171] K. Cheung and T.-C. Yuan, JHEP 0703, 120 (2007), arXiv:hep-ph/0701107 [hep-ph]

[172] D. Feldman, Z. Liu and P. Nath, AIP Conf.Proc. 939, 50 (2007), arXiv:0705.2924 [hep-ph]

[173] S. Cassel, D. Ghilencea and G. Ross, Nucl.Phys. B827, 256 (2010), arXiv:0903.1118 [hep-ph]

[174] Y. Mambrini, JCAP 0912, 005 (2009), arXiv:0907.2918 [hep-ph]

[175] D0 Collaboration Collaboration (V. M. Abazov et al.), Phys.Lett. B695, 88 (2011), arXiv:1008.2023 [hep-ex]

[176] CMS Collaboration Collaboration (S. Chatrchyan et al.), Phys.Lett. B714, 158 (2012), arXiv:1206.1849 [hep-ex].

[177] ATLAS Collaboration Collaboration (G. Aad et al.) (2012), arXiv:1208.0949 [hep-ex]

[178] C. Campagnani, Cms collaboration talk (SUS $\overline{Y 2012, ~ B e i j i n g, ~ 2012) . ~}$

[179] H. Baer, V. Barger, A. Lessa and X. Tata (2012), arXiv:1207.4846 [hep-ph]

[180] V. D. Barger, M. Berger, J. Gunion and T. Han, eConf C010630, E110 (2001), arXiv:hep-ph/0110340 [hep-ph].

[181] S. Bogacz, J. Ellis, L. Lusito, D. Schulte, T. Takahashi et al. (2012), arXiv:1208.2827 [physics.acc-ph]

[182] D. Feldman, Z. Liu and P. Nath, Phys.Rev.Lett. 99, $25 \overline{1802}$ (2007), arXiv:0707.1873 [hep-ph]

[183] P. Nath, B. D. Nelson, H. Davoudiasl, B. Dutta, D. Feldman et al., Nucl.Phys.Proc.Suppl. 200-202, 185 (2010), arXiv:1001.2693 [hep-ph] 